\title{
GEOQUÍMICA Y GEOCRONOLOGÍA DE LAS ROCAS VOLCÁNICAS BÁSICAS Y EL GABRO DE ALTAMIRA, CORDILLERA OCCIDENTAL (COLOMBIA): REGISTRO DE AMBIENTES DE PLATEAU Y ARCO OCEÁNICO SUPERPUESTOS DURANTE EL CRETÁCICO
}

\author{
Juan Pablo Zapata-Villada ${ }^{1,2^{*}}$; Jorge Julián Restrepo ${ }^{1}$; Agustín Cardona-Molina ${ }^{1}$; Uwe Martens ${ }^{3}$ \\ DOI: http://dx.doi.org/10.18273/revbol.v39n2-2017001 @) (i) \\ Forma de citar: Zapata-Villada, J.P., Restrepo, J.J., Cardona-Molina, A., y Martens, U. 2017. Geoquímica y geocronología de las rocas \\ volcánicas básicas y el Gabro de Altamira, Cordillera Occidental (Colombia): Registro de ambientes de Plateau y arco oceánico superpuestos \\ durante el cretácico. Boletín de Geología, 39(2): 13-30.
}

\section{RESUMEN}

Modelos paleogeográficos regionales han relacionado las características temporales y composicionales del registro magmático Cretácico de la Cordillera Occidental Colombiana con ambientes contrastantes de plateau y arco oceánico, los cuales harían parte de la placa Caribe que habrían colisionado con la margen continental Suramericana durante el Cretácico Superior-Paleoceno. Nuevos datos geoquímicos y geocronológicos obtenidos en una secuencia de lavas y rocas piroclásticas de composición basáltica, intruidos por cuerpos gabróicos en la región de Altamira sugieren la existencia de un volcanismo con afinidad E-MORB formado en condiciones submarinas a subaéreas. Estas secuencias de rocas pueden ser correlacionadas con unidades de la Cordillera Occidental colombiana, los Andes de Ecuador y la margen caribeña de Suramérica. Estas rocas harían parte de un plateau oceánico formado en el Cretácico Temprano, posiblemente en una latitud más sur que la posición actual y que habría hecho parte de la placa del Caribe. Estas rocas con afinidad de plateau oceánico habrían sido posteriormente intruidas por un magmatismo de composición gabróica con edades de ca. $88 \mathrm{Ma}$, el cual presenta herencias entre $95 \mathrm{Ma}$ y $114 \mathrm{Ma}$, que posiblemente reflejen la edad del basamento en el cual se emplazaron. Este magmatismo presenta características afines a un ambiente de arco magmático y podría ser correlacionada composicional y temporalmente con otros cuerpos plutónicos tonaliticos y gabróicos de la Cordillera Occidental colombiana, en el Ecuador y del Caribe los cuales representarían un arco oceánico formado sobre la secuencia de Plateau. Las características temporales y composicionales de estas rocas sugieren que el episodio de formación de la provincia de Plateau expuesta en el occidente colombiano sería más antiguo de lo tradicionalmente considerado y que previo a la colisión con la margen continental un arco oceánico habría sido desarrollado lejos del continente, el cual habría sido activo al menos hasta los $88 \mathrm{Ma}$.

Palabras clave: $\mathrm{U}-\mathrm{Pb}$, Cordillera occidental, plateau oceanico, Plutones gabróicos, Altamira.

\section{GEOCHEMISTRY AND GEOCHRONOLOGY OF THE BASIC VOLCANIC AND GABROIC ROCKS IN THE ALTAMIRA REGION, WESTERN CORDILLERA OF COLOMBIA: A RECORD OF OVERIMPOSED CRETACEOUS PLATEAU AND OCEANIC ARC ENVIROMENTS}

\begin{abstract}
Regional paleogeographic models based on limited geochronological and geochemical data from the Western Cordillera of the Colombian Andes have suggest that the exposed Cretaceous magmatic rocks were formed in plateau and intra-oceanic arc environments before their Late Cretaceous-Paleocene accretion to the continental margin. New geochemical and geochronological constraints from volcanic, pyroclastic and intrusive plutonic rocks of the Altamira section in the northern segment of the Western Cordillera record the existence of a former basaltic sequence characterized by an E-MORB signature erupted in a subaqueous to subaerial environment. Such sequence can be correlated to other Early Cretaceous volcanic rocks in the Western Cordillera of Colombia, that together with other exposures in the Ecuadorian Andes and the Caribbean are part of a major oceanic plateau that was formed in the southwestern Pacific region that formed the allochthonous Caribbean plate. This sequence was intruded by gabbroic plutons with a tholeiitic arc affinity signature, that together with other plutons of the Western Cordillera record the formation of an oceanic arc by ca. $88 \mathrm{Ma}$, as suggested by the new U-Pb LA-ICP-MS zircon ages. Older inherited zircon ages of $95 \mathrm{Ma}$ and $114 \mathrm{Ma}$ found within this gabbroic body correlate with sedimentary and plutonic ages of the host basaltic rocks, confirming the Early Cretaceous timing for the plateau episode. Although more geochronological data is still need from the Western Cordillera, the available temporal and compositional constraints, including the data presented in this contribution, suggest that the timing of the plateau formation episode maybe older than previously suggest, and that an oceanic arc was formed over this plateau before its accretion to the continental margin.
\end{abstract}

Keywords: U-Pb, Western Cordillera, Oceanic Plateau, gabbroic Plutons, Altamira.

\footnotetext{
${ }^{1}$ Universidad Nacional de Colombia, Medellín, Antioquia, Colombia. (*)jpzapatav@unal.edu.co; jjrestrepo@fastmail.fm; agcardonamo@unal.edu.co

${ }^{2}$ Servicio Geológico Colombiano, Medellín, Antioquía, Colombia.

${ }^{3}$ Tectonic Analysis Ltd. Inglaterra. umartens@zoho.com
} 


\section{INTRODUCCIÓN}

Las plumas del manto y el magmatismo de los puntos calientes dan lugar a la formación de corteza oceánica engrosada en montes marinos o plateaus oceánicos (Condie and Abbott, 1999; White et al., 1999; Kerr et al., 2003), los cuales debido a su espesor cortical pueden acrecionarse a las márgenes de los continentes, constituyendo uno de los procesos de crecimiento de corteza continental más significativos desde el Arqueano (Kerr et al., 2003). Este proceso fue particularmente común durante el Cretácico en el Pacífico, cuando aparentemente existieron múltiples plumas mantélicas en los océanos (Kerr et al., 2003; Greene et al., 2010) y algunos de estos elementos fueron modificados por la construcción de arcos magmáticos y su posterior acreción a las márgenes continentales (White et al., 2003; Whattam and Stern, 2015).

Desde el sur de Ecuador hasta el norte en las Antillas Menores, incluyendo la Cordillera Occidental colombiana se encuentran expuestos una serie de fragmentos de rocas volcánicas, en menor medida rocas sedimentarias asociadas y algunos cuerpos de gabros, relacionadas a partir de sus características geoquímicas y distribución, como parte de un plateau oceánico, el cual se habría modificado por la construcción de arcos de islas oceánicos en sus márgenes (White et al., 1999; Van der Lelij et al., 2010; Vallejo et al., 2006; Villagómez et al., 2011).
Basados en datos geoquímicos locales, reconstrucciones paleogeográficas regionales, estos fragmentos de corteza oceánica han sido consideradados como segmentos de la Placa del Caribe, la cual habría sido formada en el Pacífico suroriental, y luego migrado hasta su posición actual, colisionando con la margen continental suramericana (Nivia, 1987, 1989, 1996; Kerr et al., 1997; Meschede and Frisch, 1998; Pindell et al., 2005; Pindell and Kennan, 2009; Villagómez et al., 2011).

AlOccidente del Sistema de Fallas Cauca-Romeral (SFCR), en el dominio volcánico oceánico, los rasgos geoquímicos de las rocas basálticas y algunas edades absolutas obtenidas en rocas gabróicas y tonalíticas (FIGURA 1), así como las edades fosilíferas en sedimentos pelágicos asociados a las rocas volcánicas, han sugerido que la formación de las rocas de plateau oceánico se extenderían entre el Barremiano y el Cenomaniano (Villagómez et al., 2011; Rodríguez y Arango, 2013; Weber et al., 2015), mientras que el arco magmático que se construye sobre el sustrato de plateau oceánico (Vallejo et al., 2006; Villagómez, 2010; Van der Lelij et al., 2010) habría comenzado a formarse después de 90 Ma (Villagómez et al., 2011; Weber et al., 2015). Este registro temporal para la historia de la formación del Plateau oceánico del Caribe y el arco oceánico asociado es más antiguo que las edades entre 91 y 88 Ma sugeridas por otros autores como parte de la fase principal (Kerr et al., 1997; Sinton et al., 1998; Hauff et al., 2000; Révillon et al., 2000; Hoernle et al., 2004).
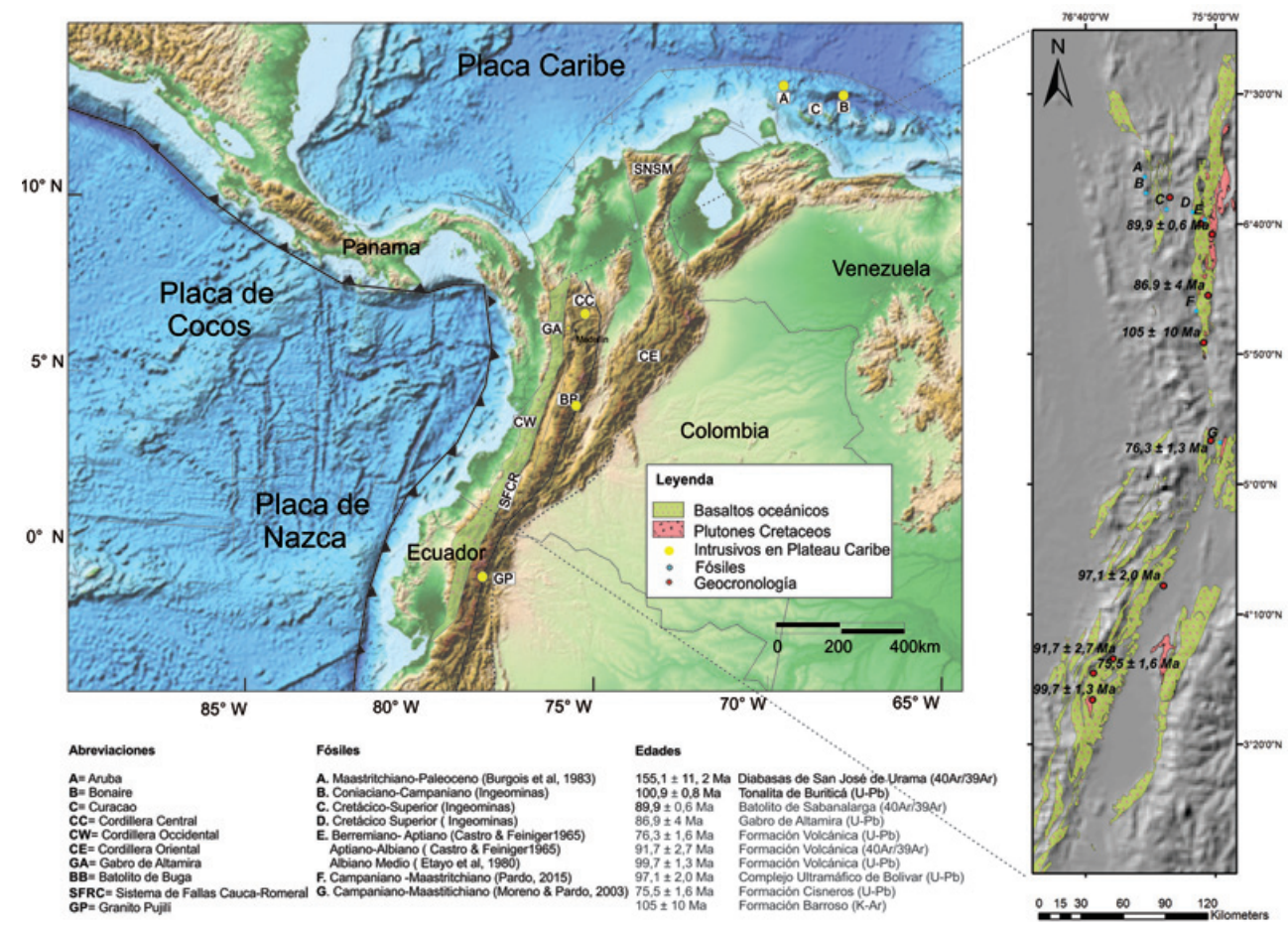

FIGURA 1. Modelo de Elevación digital para el Caribe y el norte de Sudamérica (Fuente: Amante y Eakins, 2009), edades bioestratigráfícas, geocronología en la Cordillera Occidental Colombiana. 
En este trabajo se presenta la cartografía, petrografía, geoquímica y geocronología U-Pb (LA-ICP-MS) de una sección geológica localizada en el flanco oriental de la Cordillera Occidental, en el corregimiento de Altamira, Antioquia (FIGURA 2 y 3). Esta sección expone de manera relativamente continua una sucesión de rocas volcánicas y plutónicas que inicialmente por la presencia de rocas piroclásticas habría sido relacionada con un arco magmático (Ramírez, 1976; Restrepo y Toussaint, 1976). Los resultados geoquímicos aquí presentados sugieren que las rocas volcánicas corresponden a una secuencia alternante de derrames lávicos y piroclástos afín a un plateau oceánico, intruidas por un pluton gabróico con una edad de 88 Ma y que representaría las fases iniciales de evolución de un arco magmático toleítico intraoceánico.

Estos resultados confirmarían que los fragmentos de plateau oceánico expuestos en los Andes Colombianos son efectivamente más antiguos que el Campaniano, e indicarían que la acreción de estas unidades oceánicas al continente habría ocurrido después de 86 Ma.
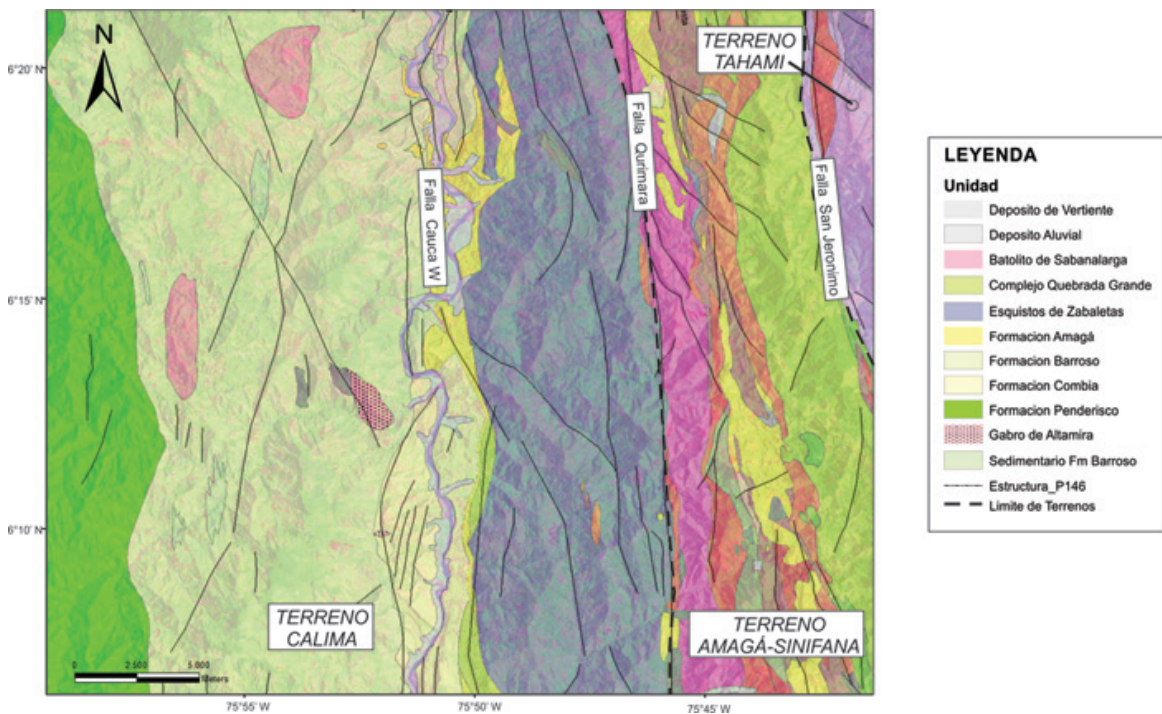

FIGURA 2. Mapa regional modificado de Mejía et al. (1983), límite oriental Falla Quirimará.
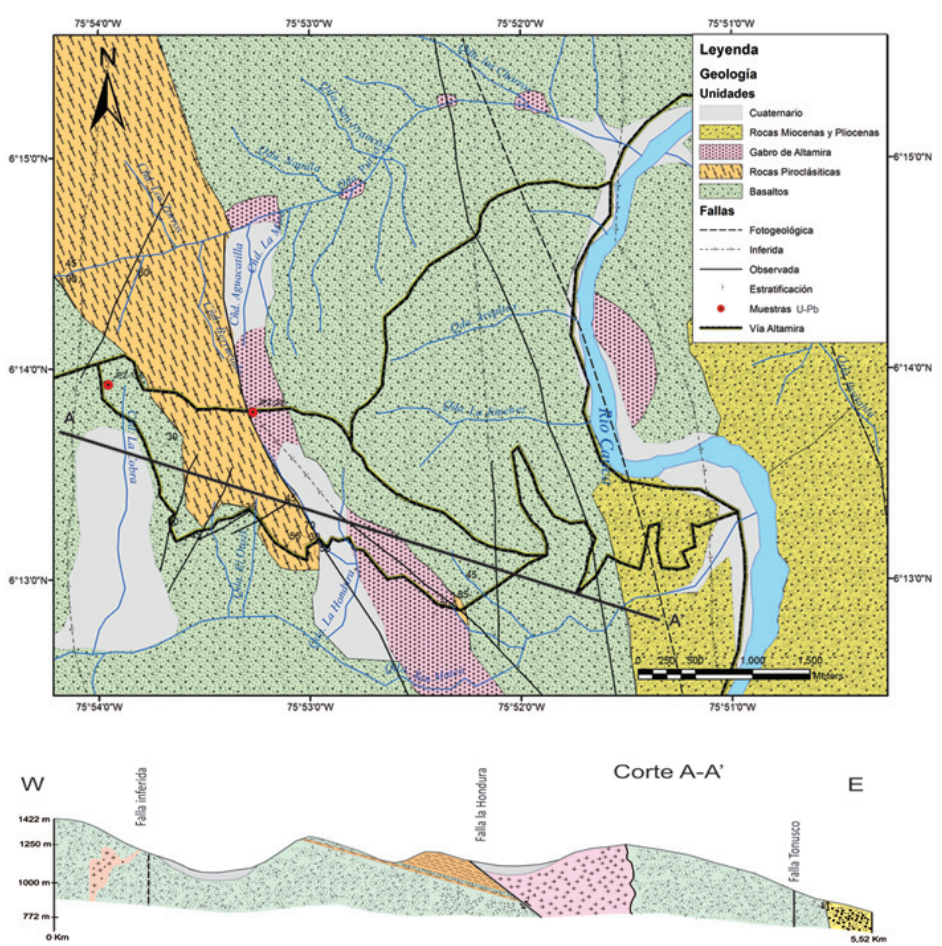

FIGURA 3. Mapa geología local y perfil geológico de Altamira, Antioquia. 


\section{MARCO GEOLÓGICO}

El borde de Placa Suramericana y la cadena Andina en los Andes de Colombia está compuesto por un mosaico de terrenos de afinidades oceánicas acrecionados a la corteza continental desde el Cretácico Superior (Case et al., 1971; Meissner et al., 1976; Duque-Caro, 1984; Restrepo and Toussaint, 1988; Kerr et al., 1997; Cediel et al., 2003; Cardona et al., 2010; Villagómez et al., 2011). Estos terrenos de afinidad oceánica que constituyen la región del ante-arco Pacífico, la Cordillera Occidental de Colombia y Ecuador, e incluyen la parte más occidental de la Cordillera Central colombiana, limitados al Este por el SFCR, que forma un cordón de franjas limitadas por fallas anastomosadas de continuidad regional hasta el Golfo de Guayaquil en Ecuador, en la cual se mezclan litologías de diferente origen y edad (Barrero, 1979; Restrepo and Toussaint, 1988) (FIGURA 1), y la cual los separa del terreno Tahamí de afinidad continental que constituye gran parte de la Cordillera Central, y un basamento metamórfico Paleozoico y Triásico y el terreno Amagá-Sinifana compuesto de franjas obduccidas al continente (Restrepo et al., 2009) (FIGURA 2).

Las rocas de afinidad oceánica al occidente del SFCR y que forman gran parte de la Cordillera Occidental (Terreno Calima), están compuestas por basaltos, sedimentos de carácter químico y siliciclástico, y rocas piroclásticas denominados al norte de la cordillera Occidental como Formación Barroso (Álvarez y González, 1978) y al sur como el Grupo Diabásico (Barrero, 1979) y Formación Volcánica (Aspden, 1984). Estas unidades son intruidas por una serie de plutones de gabro y tonalita (Restrepo y Toussaint, 1976; González et al., 1978; Villagómez et al., 2011).

La Formación Penderisco constituida por areniscas con alto contenido de cuarzo y micas, intercaladas con lodolitas, o constituida por intercalaciones de cherts y lodolitas reposan discordantemente sobre rocas volcánicas de la Formación Barroso (Álvarez y González, 1978) expuestas como franjas delgadas.

El origen oceánico y la acreción de las rocas volcánicas expuestas en la Cordillera Occidental colombiana se remonta a la década del 70 (Case et al., 1971; Meissner et al., 1976; Toussaint y Restrepo, 1976; Barrero, 1979; Feininger et al., 1972). Estos autores sugirieron inicialmente que estas rocas podrían estar relacionadas con ambientes de corteza oceánica o con arcos de islas. Posteriormente Nivia $(1987,1989,1996)$ y Kerr et al. (1997) obtienen datos geoquímicos en estas secuencias y sugieren una correlación con ambientes de punto caliente como el de Islandia y correlacionan estas rocas con otras unidades volcánicas Cretácicas del circum-caribe, indicando que en conjunto hacen parte de un plateau oceánico formado a partir de una pluma mantélica.

Recientemente, Rodríguez y Arango (2013) presentan nuevos datos geoquímicos en las rocas volcánicas y sugieren que las unidades volcánicas básicas de la Cordillera Occidental incluyen también rocas formadas en ambiente de arco. Mientras que las características geoquímicas de los plutones tonalíticos del Cretácico Tardío que intruyen estas secuencias volcánicas han sido relacionados con ambientes de arco de islas oceánico el cual se habría construido sobre las rocas formadas previamente en el ambiente de plateau oceánico (Villagómez et al., 2011; Weber et al., 2015).

Las rocas gabroicas asociadas a las secuencias basálticas han sido relacionadas en general con los niveles estructurales más profundos de las secuencias de plateau (Nivia, 1996), y algunas de ellas han arrojado edades Cretácicas de ca. $98 \mathrm{Ma}$. Sin embargo como será discutido en esta contribución, los plutones gabroicos que cortan la secuencia basáltica de plateau incluyen diferentes tipos composicionales y registran ambientes tectónicos contrastantes, como es el caso del Gabro de Altamira que será discutido en esta contribución.

Dentro del SFCR aparecen bloques exóticos de afinidad de arco y edad Cretácica agrupadas dentro del Complejo Quebradagrande, rocas metamórficas formadas en complejos de subducción/acreción del Complejo Arquía, y rocas de afinidad oceánica del Grupo Amaime, cubiertas por sedimentos marinos a transicionales de la Formación Nogales (Maya y González, 1995; Nivia, 1996; Toussaint y Restrepo, 1974; Aspden, 1984; Moreno-Sánchez and Pardo-Trujillo, 2003).

Al Occidente del SFCR, la Cordillera Occidental de Colombia se encuentra constituida por rocas de afinidad oceánica que han sido incluidos dentro del Terreno Cuna y Calima (Restrepo and Toussain, 1988). El terreno Calima está limitado al Este por el SFCR, que y al Oeste por el sistema de Fallas de Dabeiba que los separa del terreno Cuna (Restrepo and Toussaint, 1988) con características de arco de islas oceánico y afinidad toleítica con edades Eoceno - Paleoceno (Duque-Caro, 1989; Kerr et al., 1997).

El ambiente donde se originó la Formación Barroso es todavía debatido. Para algunos autores esta secuencia presenta afinidad con la Fm. Volcánica (Aspden, 1984) al sur de Colombia, con un carácter geoquímico afín con un plateau oceánico (Kerr et al., 1997, 2003; Villagómez, 
2010). Sin embargo otros autores presentan evidencias de que las rocas volcánicas de la Fm. Barroso pueden incluir elementos de plateau, MORB y de arco de islas cuya relación genética podría ser parte de una historia de superposición de ambientes tectónicos o simplemente representar elementos estructuralmente contrastantes (Restrepo y Toussaint, 1976; Álvarez y González, 1978; Parra, 1978; González, 2001; Villagómez, 2010; Rodríguez y Arango, 2013).

A raíz de estos contrastes, Rodríguez y Arango (2013) sugieren incorporar una nueva nomenclatura estratigráfica, en la cual las rocas con características geoquímicas que se puedan correlacionar con el plateau oceánico que conforma el occidente Colombiano se denominen San José de Urama, y las rocas con afinidad de arco corresponderían a la Formación Barroso.

Dado que los límites de estas dos unidades no están claramente definidos, en esta contribución mantenemos la terminología tradicional adoptada por Kerr et al. (1997).

Edades K-Ar en RT (Roca total) de 105 \pm 10 Ma (Toussaint y Restrepo, 1978) y Ar-Ar en RT de 84,2 $\pm 1,4$ Ma (Geoestudios-Ingeominas, 2005), han sido reportadas para la Fm. Barroso cerca de su sección tipo en Antioquia. Mientras que en el sur de Colombia, para la Fm. Volcánica se reportan edades Ar-Ar de 92 Ma (Kerr et al., 1997) en doleritas y edades U-Pb de 98 Ma para el Gabro del Palmar (afinidad de Plateau) y de 92 Ma para el Batolito de Buga, los cuales intruyen las rocas basálticas con afinidad de Plateau (Villagómez et al., 2011).

Las rocas sedimentarias hemipelágicas y terrígenas asociadas a estas unidades volcánicas en el Norte de la Cordillera Occidental presentan edades bioestratigráficas que van desde el Barremiano hasta el Aptiano (González, 2001). Mientras que las secuencias siliclásticas, que incluyen intercalaciones de areniscas y lodolitas con material cuarzoso de afinidad continental, presentan edades entre los intervalos: Aptiano -Albiano (Etayo-Serna, 1989), y Santoniano- Mastrichiano (Moreno-Sánchez and Pardo-Trujillo, 2003) y cubren las rocas volcánicas, limitando su acreción al continente (FIGURA 1).

A pesar de los pocos datos geocronológicos existentes y que posiblemente el significado de las edades Ar-Ar esté comprometido por el bajo potasio y la alteración hidrotermal que pueden tener las rocas volcánicas, es posible inferir una edad mínima para la Formación Barroso, con base en las edades paleontológicas del Cretácico inferior de las rocas sedimentarias con las que está intercalada y a la relación intrusiva con rocas plutónicas fechadas entre $84,97 \pm 2,5$ Ma a $100 \mathrm{Ma}$ (Ordóñez-Carmona et al., 2003; Weber et al., 2015).

Estas secuencias volcánicas de la Formación Barroso han sido asociadas a un ambiente de plateau y a arcos relacionados con la formación y migración de la placa del Caribe desde el Pacífico (Kerr et al., 1997). Placa que se habría formado como parte de un plateau oceánico que habría hecho erupción entre 88 y $100 \mathrm{Ma}$ (Kerr et al., 1997, 2009; Hauff et al., 2000; Thompson et al., 2004; Villagómez et al., 2011; Wright and Wyld, 2011) o a subducción de una dorsal oceánica (Serrano et al., 2011), y luego intruído por una serie de cuerpos de afinidad calco alcalino a lo largo del Caribe como el Batolito de Buga, Sabanalarga, Tonalita de Buriticá, Granito Pujilí en Ecuador y Batolito de Aruba entre 90 y 80 Ma (Kerr et al., 1997, 2009; Hauff et al., 2000; Thompson et al., 2004; Van der Lelij et al., 2010; Villagómez et al., 2011; Wright and Wyld, 2011). Posteriormente este plateau habría colisionando con la margen Suramericana aproximadamente entre 75 y $73 \mathrm{Ma}$, dejando los fragmentos presentes en los Andes Colombianos, Ecuatorianos y en islas a lo largo del Caribe (Villagómez, 2010; Spikings et al., 2015).

\section{METODOLOGÍA}

El trabajo de campo se realizó en el flanco oriental de la Cordillera Occidental en la región de Altamira corregimiento de Betulia, Antioquia. Para su elaboración se revisó la cartografía realizada por Ramírez (1976). Se cartografiaron las unidades principales a escala 1:25.000 y se tomaron muestras de roca para análisis petrográficos, geoquímicos y de geocronología de $\mathrm{U}-\mathrm{Pb}$ en circón.

\section{Geoquímica de Roca total}

Las muestras seleccionadas para elementos mayores fueron analizadas en Actlabs mediante un espectrómetro de masa con plasma inductivamente acoplado (ICP) y en el Laboratorio Universitario de Geoquímica Isotópica (LUGIS), en la Universidad Nacional Autónoma de México (UNAM), Ciudad Universitaria, Ciudad de México, mediante un equipo de Fluorescencia de rayos X (FRX) Siemens SRS-3000. Los análisis fueron realizados sobre las muestras pulverizadas siguiendo procedimientos descritos por Lozano-Santacruz et al. (1995) y Verma et al. (1996). Los elementos trazas fueron obtenidos por medio de espectrometría de masas en dos laboratorios diferentes ambos con plasma inductivamente acoplado (Inductively Coupled Plasma Mass Spectrometry, ICP-MS). Cuatro muestras fueron 
analizadas en los laboratorios de Actlabs y tres muestras en el LEI (Laboratorio de Estudios Isotópicos), UNAM, Campus Juriquilla, usando un equipo Thermo Series XII, según procedimientos descritos por Mori et al. (2007). Para la calibración del equipo se emplearon los estándares JB-2, AGV-2, BCR-2, BHVO-2, JR-1 y ZZ.

\section{Geocronología U-Pb LA-ICP-MS}

El análisis de las muestras fue realizado en las instalaciones del LEI- UNAM, utilizando un sistema de ablación láser (LA) Resonetics M-50, láser $\mathrm{ArF}$ Eximer operado a $193 \mathrm{~nm}, 5 \mathrm{~Hz}$ y $\pm 8 \mathrm{~J} / \mathrm{cm}^{2}$; empleando un punto de ablación de $23 \mu \mathrm{m}$ de diámetro. Este láser se encuentra adaptado a una estación de trabajo microcontrolada, con una celda de ablación de doble volumen, la cual es llenada de He al momento de la ablación y luego un flujo de $\mathrm{N}^{2}$ y Ar transporta la muestra a un ICPMS cuadrupolo Thermo Xii series (Solari et al., 2010). La reducción de los datos de cada análisis fue realizada mediante una secuencia de comandos (Upb.age) para el programa estadístico R (Solari et al., 2010). Esta secuencia de comando o script realiza automáticamente el proceso de integración de las señales del ICP-MS y su respectiva corrección por blancos, así como el cálculo de relaciones isotópicas $\left({ }^{206} \mathrm{~Pb} /{ }^{238} \mathrm{U},{ }^{207} \mathrm{~Pb} /{ }^{206} \mathrm{~Pb}\right.$ y ${ }^{208} \mathrm{~Pb} /{ }^{232} \mathrm{Th}$ ) y sus respectivos errores a \pm 1 sigma. Con la finalidad de hacer correcciones por el fraccionamiento intrínseco de los isótopos de $\mathrm{Pb}$ y la deriva instrumental se mide por cada 5 mediciones de la muestra un circón estándar, además la secuencia de análisis incluye la medición de una muestra de referencia (vidrio NIST 612; Solari et al., 2010). En este caso se utilizaron circones del estándar Plešovice, el cual tiene una edad concordante calculada en $337,13 \pm 0,37$ Ma (2 sigma)
(Sláma et al., 2008). Los datos fueron graficados y calculadas sus respectivas edades sin corrección por $\mathrm{Pb}$ común, utilizando Isoplot 3.0 (Ludwig, 2004). El isótopo ${ }^{204} \mathrm{~Pb}$, comúnmente utilizado para correcciones por $\mathrm{Pb}$ común, no fue medido debido interferencias del isótopo ${ }^{204} \mathrm{Hg}$ que acompaña el He durante las mediciones.

\section{RESULTADOS}

\section{Relaciones de campo y muestreo}

En la región de Altamira (suroeste Antioqueño) se encuentra expuesta una sección bien preservada de basaltos almohadillados (FIGURA 4A), basaltos amígdalares, basaltos masivos a diabasas, intercalados hacia el tope con secuencias subaéreas de rocas piroclásticas, incluyendo lapilli acrecional (McPhie et al., 1993), tobas y brechas piroclásticas (FIGURAS 4D y 4E). En ocasiones se observa preservada la estratificación en los flujos de pillow lavas (FIGURA 4A). En el área de estudio la unidad aflora en un área aproximada de $11 \mathrm{~km}^{2}$ (FIGURA 3), con un espesor máximo de $700 \mathrm{~m}$. Las muestras de basaltos presentan texturas ofíticas, subofíticas microcristalinas y texturas amigadalares, los cuales se encuentran relativamente frescos, presentan en ocasiones matriz microlítica de plagioclasa albitizada y augita con amígdalas rellenas de zeolitas, cuarzo y calcita (FIGURAS 4B y 4C). Las rocas piroclásticas presentan esencialmente vidrio parcialmente palagonitizado (en forma de shards) y fragmentos de líticos basálticos (FIGURA 4F y 4G). En sección delgada en ambas unidades se evidencian minerales como prenhita rellenando zeolitas, típicos de metamorfismo de piso oceánico de facies prenhitapumpellita (Frey et al., 1991).
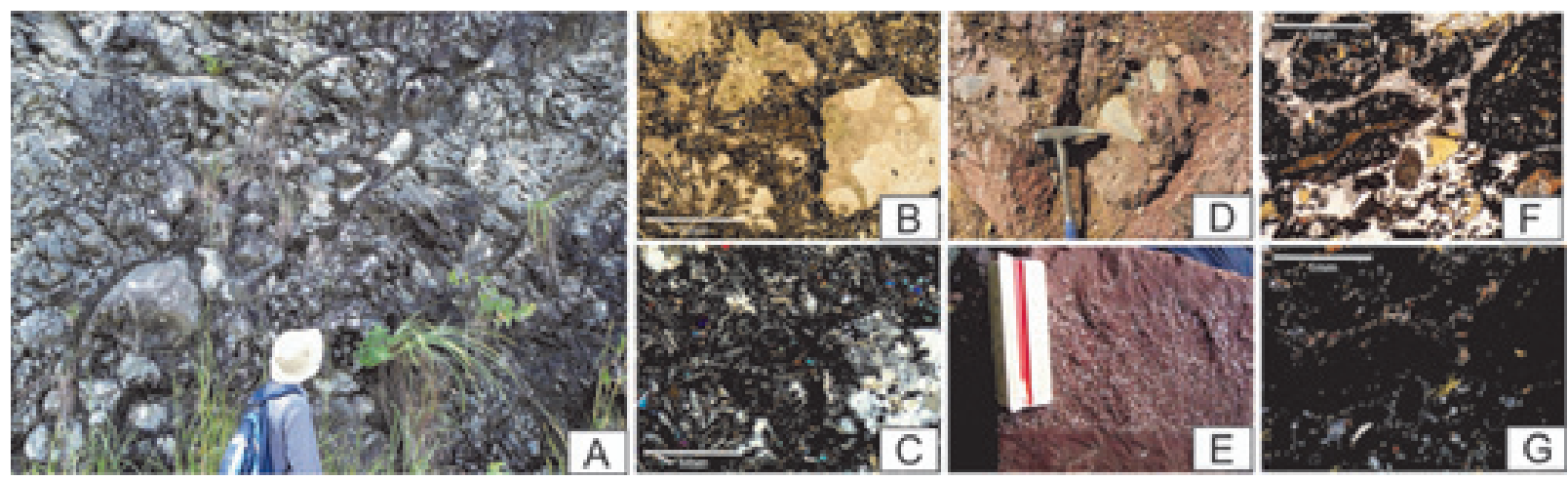

FIGURA 4. A. Pillow lavas con psudoestratificación. B. y C. Microfotografía de Basalto amígdalar de matriz microlitica. D. Aglomerado volcánico. E Toba de lapilli. F. y G. Microfotografía Lapilli acrecional con Shards de vidrio.

Estas secuencias volcánicas son intruídas por el Gabro de Altamira evidenciándose bordes de enfriamiento del gabro y contactos tajantes con las secuencias volcánicas (Ramírez, 1976). El Gabro de Altamira es un cuerpo intrusivo de $2,5 \mathrm{~km}^{2}$ (FIGURA 3) de forma elongada con dirección NW-SE. El Gabro de Altamira está compuesto principalmente de clinopiroxeno, ortopiroxeno, plagioclasa y en ocasiones hornblenda, 
y trazas de cuarzo intersticial (FIGURAS 5A, 5B y 5C). Al Este de la zona se observa intruyendo basaltos de la Fm. Barroso, mientras que al oeste se observan en contacto fallado con rocas piroclásticas (FIGURA 5A). Son comunes otros cuerpos menores de similar composición que intruyen la formación Barroso pero que no son cartografiables en la escala del trabajo, pero que presentan como característica principal una orientación N-S y una composición dioritica-gabróica.
Una unidad de andesitas porfídicas con hornblenda (FIGURA 5D) no mencionada en trabajos previos, fue encontrada en la zona de estudio. Esta unidad se encuentra intruyendo basaltos de la Fm. Barroso y el Gabro de Altamira (FIGURA 5E). En sección delgada presenta fenocristales de hornblenda (ocasionalmente reemplazados a zoisita-clinozoisita), en una matriz de plagioclasa, hornblenda y en ocasiones pirita (alterada a minerales del grupo de las arcillas) (FIGURA 5F).
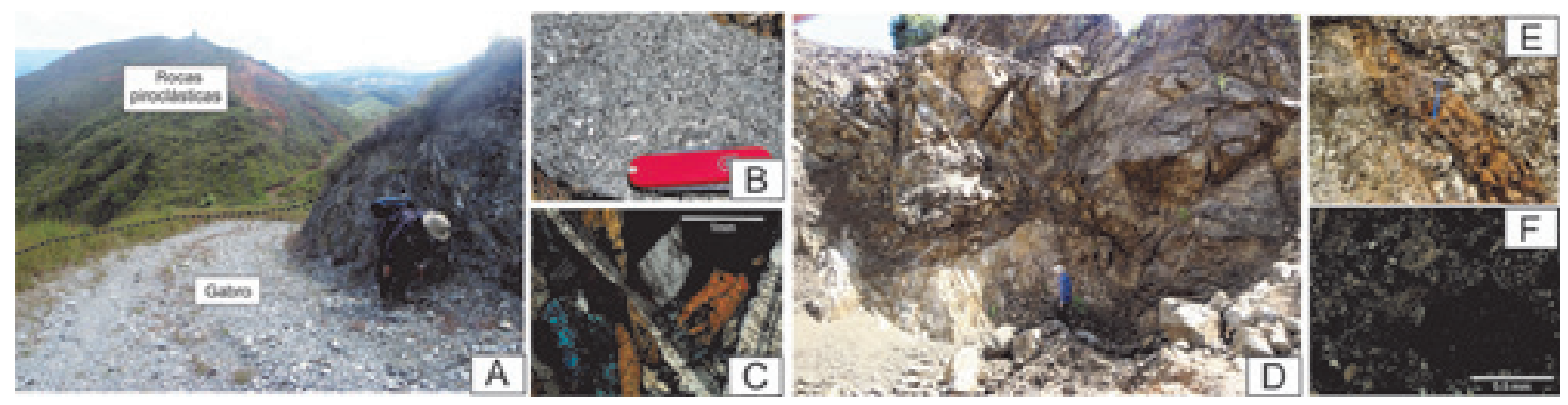

FIGURA 5. A. Contacto Fallado Piroclastos-Gabro de Altamira. B. Aspecto Macro Gabro. C. Microfotografía Gabro (Cpx+Pl). D. Intrusivos Andesíticos en Basaltos. E. Dique Andesítico en Basaltos. F. Microfotografía Andesita Hornbléndica.

\section{Geoquímica}

Se analizaron elementos mayores y trazas para 12 muestras (TABLA 1), distribuidas entre basaltos (1524, 1526 y 1527) y rocas piroclásticas (JPA 6, JPA 26-A, JPA 26-B) de la Fm. Barroso, muestras correspondientes al Gabro de Altamira (1525, JPA-29, JPA-38, JJ 1649), andesitas hipoabisales (JPA-15B, JPA-41) que intruyen la Fm. Barroso y el Gabro de Altamira. Las muestras se encuentran relativamente frescas (basaltos con pérdidas al fuego entre 2,14-1,99 y gabros entre 0,6 y 1,54), y moderadamente alteradas (andesitas entre 3,09 y 6,13 , y las rocas piroclásticas entre 4,16 y 7,28).

El contenido de $\mathrm{SiO}_{2}$ varía para las muestras de basaltos entre 47,67-50,16 $\mathrm{wt} \%$, las rocas piroclásticas entre $46,13-51,79 \mathrm{wt} \%$, los gabros entre 48,76-49,83 wt $\%$ $\mathrm{y}$ las andesitas entre 50,9-59,7 wt $\%$ confirmando la descripción de campo. El diagrama $\mathrm{Zr} / \mathrm{Ti}-\mathrm{Nb} / \mathrm{Y}$ (FIGURA 6A) basado en elementos relativamente inmóviles muestra que la mayoría de las muestras caen en el campo de basalto y/o gabros exceptuando una muestra de andesita (JPA-15B) que cae en el campo de las dacitas; esta muestra se encuentra relativamente alterada $(\mathrm{LOI}=6,13)$. La mayoría de las muestras corresponden a las series toleíticas bajas en $\mathrm{K}(<0,20)$ exceptuando la muestra JPA-15 B que cae en el campo de las series calco alcalinas (FIGURA 6B).

Diagramas multielementos normalizados al N-MORB (FIGURA 6C), el Gabro de Altamira se caracteriza por anomalías negativas de $\mathrm{Ta}, \mathrm{Nb}$ y Ti y valores de
REE relativamente planos $\left((\mathrm{La} / \mathrm{Sm})_{\mathrm{N}}=1,54-2,03 \mathrm{y}\right.$ $(\mathrm{La} / \mathrm{Yb})_{\mathrm{N}}=1,64$ a 2,07). Las muestras de andesita presentan un enriquecimiento en elementos LILE respecto HFSE, y en LREE respecto a HREE $\left((\mathrm{La} / \mathrm{Sm})_{\mathrm{N}}=3,28\right.$ a 5,32 y $(\mathrm{La} / \mathrm{Yb})_{\mathrm{N}}=6,50$ a 9,26$)$.

En el diagrama normalizado al Condrito (FIGURA 6D) se evidencian para las muestras correspondientes a basaltos, que los elementos LILE no se encuentran enriquecidos respecto a HFSE, presentando además patrones planos de tierras raras $(\mathrm{La} / \mathrm{Sm})_{\mathrm{N}}=1,85$ a 1,9 y $(\mathrm{La} / \mathrm{Yb})_{\mathrm{N}}=1,85$ a 2,0 (FIGURA 6D), las muestras correspondientes a rocas piroclásticas presentan patrones de REE similares $(\mathrm{La} / \mathrm{Sm})_{\mathrm{N}}=2,19$ a 2,84 y $(\mathrm{La} / \mathrm{Yb})_{\mathrm{N}}=2,41$ a 4,19 (FIGURA 6D).

Al igual que los gabros, las muestras de andesitas se caracterizan por presentar anomalías negativas de Ta, $\mathrm{Nb}$ y Ti siendo más fuertes para el grupo de andesitas. En el diagrama de REE normalizado al Condrito las andesitas presentan patrones enriquecidos (FIGURA 6D).

Las relaciones $\mathrm{Nb} / \mathrm{Yb}$ vs $\mathrm{Th} / \mathrm{Yb}$ muestran como los basaltos y rocas piroclásticas presentan características típicas de E-MORB y se sobreponen en el campo del Plateau Colombia-Caribe (FIGURA 6E), mientras que los gabros y las andesitas se caracterizan por presentar enriquecimientos en Th (Kerr et al., 1997).

Cuando se comparan las características geoquímicas de los gabros con otras unidades plutónicas, intrusivas en 
las rocas basálticas de la Formación Barroso (FIGURA 6E) son similares a la tonalita de Buriticá y el Batolito de Sabanalarga, localizados al norte de la Cordillera Occidental (Weber et al., 2015), los cuales han sido relacionados con procesos de fusión de la corteza de plateau durante el crecimiento del mismo, y con las fases tempranas de formación de un arco volcánico (Weber et al., 2015). Mientras que las andesitas, con valores más altos en la relación $\mathrm{Th} / \mathrm{Yb}$ y $\mathrm{Nb} / \mathrm{Yb}$ se van hacia un campo más afín con un arco magmático más maduro.
Para el Gabro de Altamira (Ordóñez-Carmona, 2001) reporta valores primitivos con $\varepsilon \mathrm{Nd}_{(\mathrm{T})}=4,78-6,58$ y relaciones ${ }^{87} \mathrm{Sr} /{ }^{86} \mathrm{Sr}=0,70393-0,70348$, mientras que para los cuerpos que intruyen la Fm. Barroso como el Batolito de Sabanalarga y el Gabro de Hispania (FIGURA 6F) se presentan valores de ${ }^{87} \mathrm{Sr} /{ }^{86} \mathrm{Sr}=$ 0,70370 y $\varepsilon \mathrm{Nd}_{(\mathrm{T})}$ cercanos a 7 , los cuales igualmente son semejantes a otras rocas con afinidad de arco magmático oceánico que igualmente intruyen rocas con afinidad de plateau oceánico como el Batolito de Aruba y el Granito Pujilí (White et al., 1999; Vallejo et al., 2006).

TABLA 1. Resultados geoquímicos de roca total.

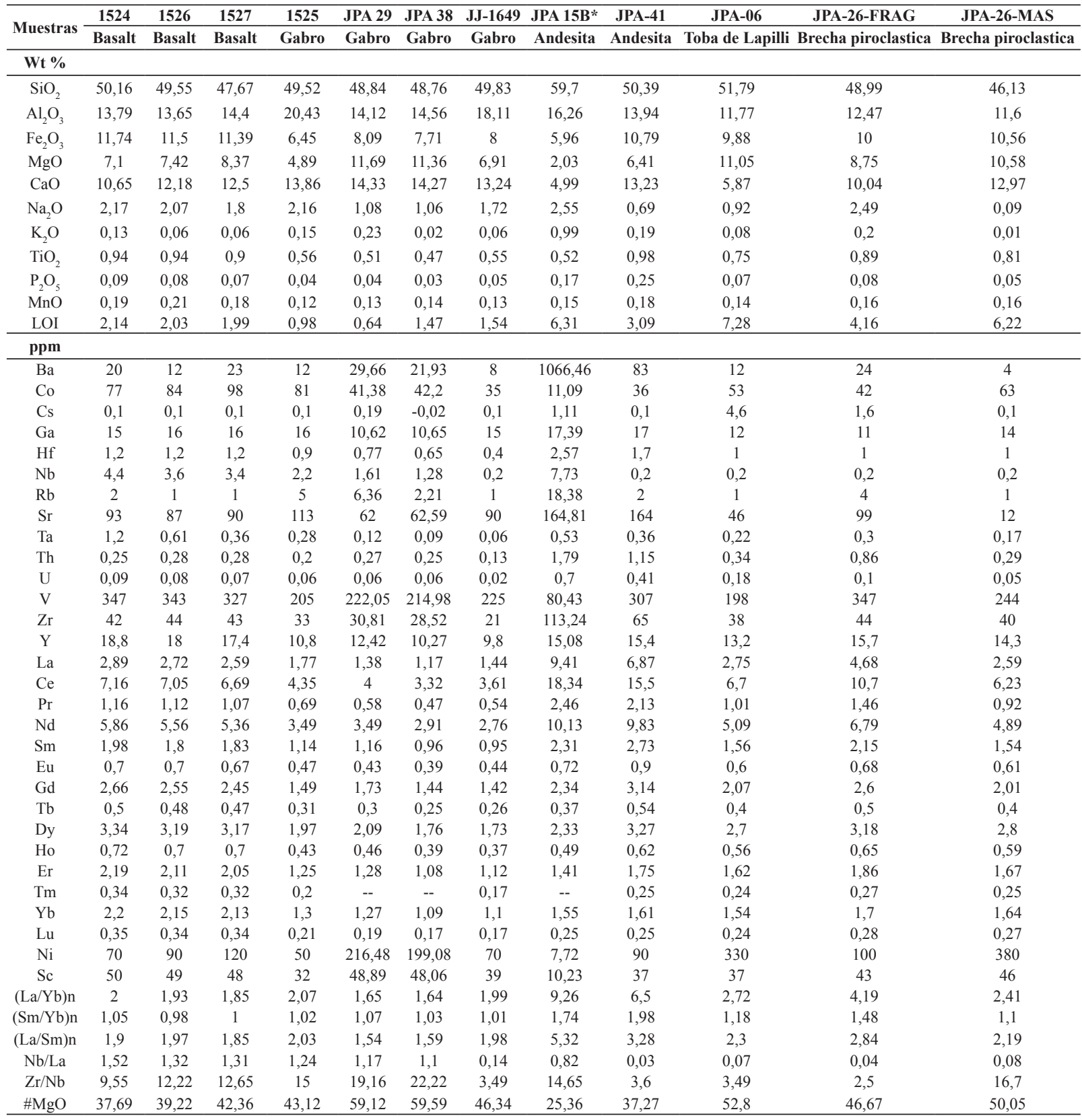

* Muestras alteradas. 

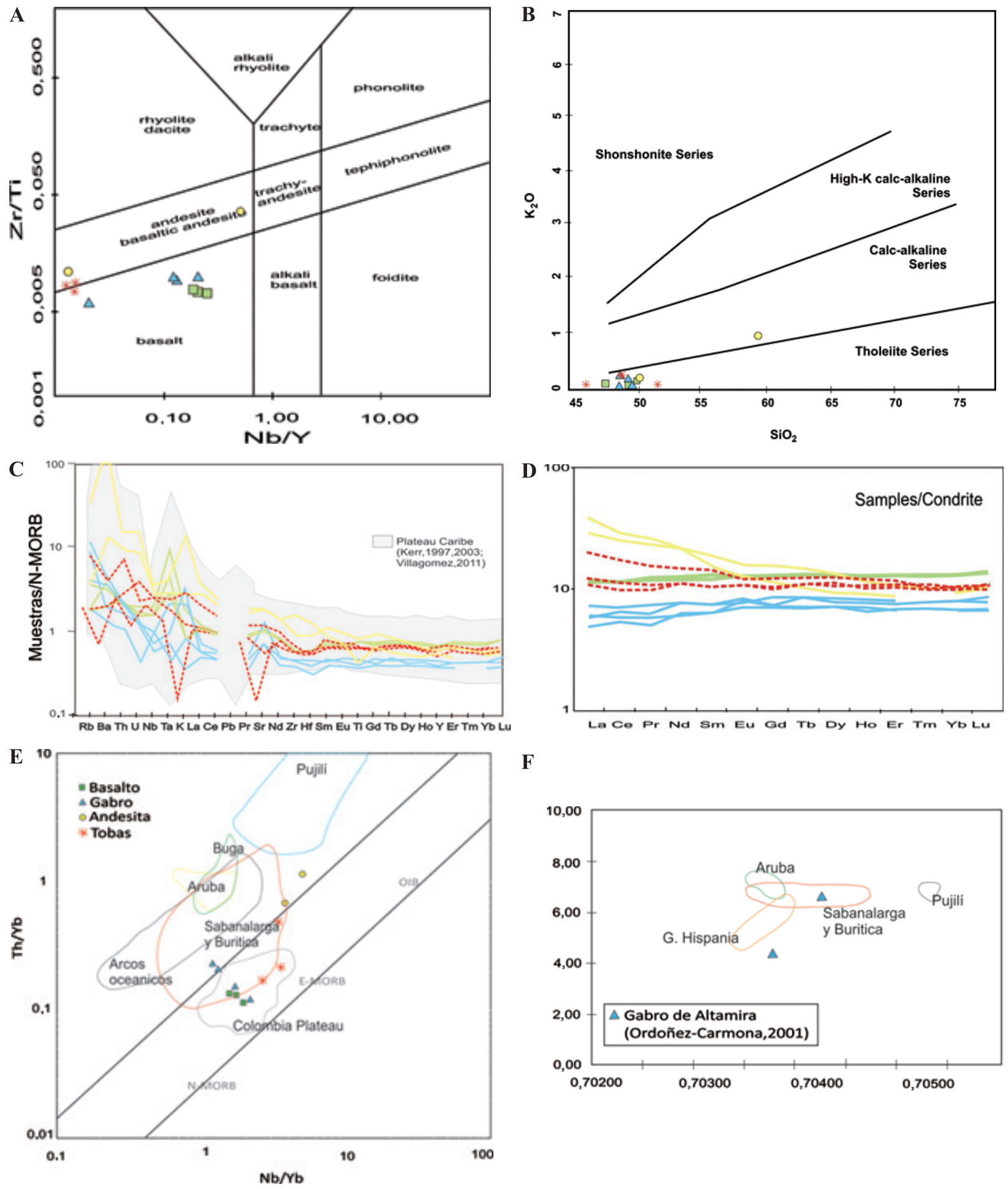

F

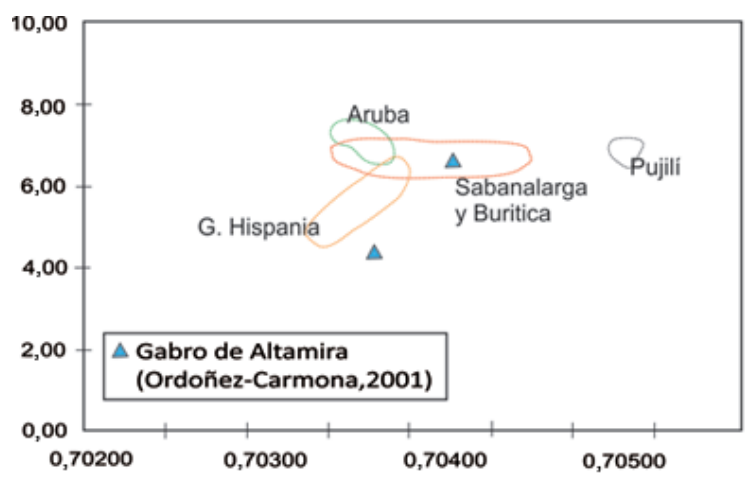

\begin{tabular}{|c|c|c|c|c|c|c|c|}
\hline \multicolumn{8}{|c|}{ Legend } \\
\hline \multicolumn{4}{|c|}{ Classification } & \multicolumn{4}{|c|}{ Spidergrams } \\
\hline$\square$ Basaltos & $\triangle$ Gabros & O Andesitas & * Tobas & Basaltos & Gabros & Andesitas & -=- Tobas \\
\hline
\end{tabular}

FIGURA 6. A. Clasificación de las muestras analizadas, según diagramas de Winchester y Floyd (1977). B. Clasificación de las muestras analizadas, según diagramas de Peccerillo y Taylor (1976). C. Diagrama multielementos normalizado al N-MORB (Sun and McDonough, 1989). D. Diagrama multielementos normalizado al Condrito (Nakamura, 1974). E. Diagrama Th/Yb$\mathrm{Nb} / Y b$. (Pearce, 2008). F. $\varepsilon N d y{ }^{87} \mathrm{Sr} /{ }^{86} \mathrm{Sr}$ para el Gabro de Altamira (Ordoñez-Carmona, 2001; Weber et al., 2015). 


\section{Geocronología}

Dos muestras fueron procesadas para ser analizadas por el método U-Pb (LA-ICP-MS), de cada una fueron obtenidas imágenes de catodoluminiscencia (CL, FIGURA 7). Algunos cristales presentan zonación, aunque en la mayoría de los cristales es escasa y es típico encontrar texturas de reabsorción magmática.

Los datos analíticos de las dos muestras son mostrados en la TABLA 2. En ambas muestras todos los circones presentan relaciones $\mathrm{Th} / \mathrm{U}$ entre 0,63 y 12,76 características de procesos magmáticos (Rubatto, 2002).

Cuarenta circones fueron separados de una muestra del Gabro de Altamira (JPA-29) con coordenadas $6^{\circ} 13^{\prime} 27.4^{\prime \prime} \mathrm{N}$ y $75^{\circ} 52^{\prime} 58.3^{\prime \prime} \mathrm{W}$. Los circones de esta muestra presentan relaciones $\mathrm{Th} / \mathrm{U}$ entre 0,1 y 5,49 , características de circones magmáticos (Rubatto, 2002).

Los resultados de la muestra JPZ-029 se graficaron en un diagrama Tera y Wasserburg (1972) donde se calculó una edad media ponderada de $87,67 \pm 0,90 \mathrm{Ma}$ interpretada como edad de cristalización (FIGURA 7), con un MSWD de 3,7 y $\mathrm{n}=18$, las elipses de error son graficadas a $\pm 2 \sigma$. En la muestra además se observaron dos grupos de datos más antiguos con medias de $95 \pm 1,2$ Ma y $114 \pm 15$ Ma asociados con edades de posibles cristales heredados del basamento cretácico.

Adicionalmente un cristal de circón obtenido de una muestra de andesita porfídica (JPA 15B), ubicado en 6 13 '39" N y 75'53'43" W fue analizado, y arrojó una edad ${ }^{206} \mathrm{~Pb} /{ }^{238} \mathrm{U}$ de $86,9 \pm 4 \mathrm{Ma}$.

TABLA 2. Resultados de los análisis geocronológicos U-Pb en circones.

\begin{tabular}{|c|c|c|c|c|c|c|c|c|c|c|c|c|c|c|c|c|c|c|c|}
\hline \multirow[b]{2}{*}{ Analysis } & \multirow[b]{2}{*}{$\underset{(\mathbf{p p m})^{1}}{\mathrm{U}}$} & \multirow[b]{2}{*}{$\begin{array}{c}\text { Th } \\
(\mathbf{p p m})^{1}\end{array}$} & \multirow[b]{2}{*}{ Th/U } & \multicolumn{8}{|c|}{ CORRECTED RATIOS $^{2}$} & \multirow[b]{2}{*}{ Rho } & \multicolumn{7}{|c|}{ CORRECTED AGES (Ma) } \\
\hline & & & & ${ }^{207} \mathrm{~Pb} /{ }^{206} \mathrm{~Pb}$ & \pm 2 abs & $\mathbf{P b} /{ }^{235} \mathbf{U}$ & \pm 2 abs & ${ }^{206} \mathbf{P b} /{ }^{238} \mathbf{U}$ & \pm 2 abs & ${ }^{208} \mathrm{~Pb} /{ }^{232} \mathrm{Th}$ & \pm 2 abs & & ${ }^{206} \mathrm{~Pb} /{ }^{238} \mathrm{U}$ & \pm 2 & ${ }^{207} \mathrm{~Pb} / 235 \mathrm{U}$ & \pm 2 & $\begin{array}{c}\text { Best age } \\
\text { (Ma) }\end{array}$ & \pm 2 & $\begin{array}{c}\text { Disc } \\
\%\end{array}$ \\
\hline Zircon_01 & 518 & 517 & 79 & 0,046124 & 0,000034 & 0,091 & 0,0019 & 0,01359 & 0,00034 & 0,00405 & 0,00024 & 0,98 & 87 & 2,2 & 92,8 & 1,8 & 87 & 2,2 & 6,25 \\
\hline Zircon_02 & no value & no value & & 0,0460756 & 0,0000022 & 0,0955 & 0,0051 & 0,01432 & 0,00077 & 0,00387 & 0,00019 & 1 & 91,6 & 4,9 & 97,2 & r & 91,6 & 4,9 & 5 \\
\hline Zircon_03 & 227,3 & 1290 & 5,68 & 0,046096 & 0,000015 & 0,0967 & 0,0018 & 0,01452 & 0,00036 & 0,00418 & 0,00029 & 0,95 & 92,9 & 2,3 & 98,4 & 1,8 & 92,9 & 2,3 & 5 \\
\hline Zircon_04 & 475 & 1423 & 3 & 0,04671 & 0,00086 & 0,0922 & 0,0014 & 0,01391 & 0,00022 & 0,00419 & 0,00027 & 0,82 & 89,02 & 1,4 & 94,1 & 1,3 & 89 & 1,4 & 5 \\
\hline Zircon_05 & 326,4 & 2095 & 6,42 & 0,04646 & 0,00065 & 0,0942 & 0,0024 & 0,01407 & 0,0004 & 0,00394 & 0,00026 & 0,97 & 90 & 2,5 & 95,9 & 2,3 & 90 & 2,5 & 6 \\
\hline Zircon_06 & 687 & 744 & 1,08 & 0,0469 & 0,00068 & 0,0931 & 0,0023 & 0,01393 & 0,00035 & 0,00415 & 0,00031 & 0,93 & 89,2 & 2,2 & 94,9 & 2,3 & 89,2 & 2,2 & 6 \\
\hline Zircon_08 & 56 & 366 & 6,54 & 0,0460748 & 0,0000029 & 0,111 & 0,0066 & 0,01664 & 0,001 & 0,00362 & 0,00034 & 1 & 106,4 & 6,4 & 112,1 & 6,4 & 106 & 6,4 & 5 \\
\hline Zircon_09 & 225,6 & 3580 & 15,87 & 0,0460846 & 0,0000056 & 0,1028 & 0,0026 & 0,01547 & 0,00046 & 0,0041 & 0,0002 & 1 & 98,9 & 2,9 & 104,3 & 2,5 & 98,9 & 2,9 & 5 \\
\hline Zircon_10 & 678,4 & 354 & 0,52 & 0,0477 & 0,0012 & 0,0935 & 0,0016 & 0,01393 & 0,00026 & 0,00413 & 0,00055 & 0,81 & 92 & 1,6 & 95,2 & 1,5 & 89,2 & ,6 & 6 \\
\hline Zircon_11 & 563 & 830 & 1,47 & 0,046116 & 0,000028 & 0,0906 & 0,0019 & 0,01355 & 0,00033 & 0,00403 & 0,00019 & 0,99 & 8 & 2,1 & 92,5 & 1,8 & 86,8 &, 1 & 6 \\
\hline Zircon_12 & 792 & 777 & 0,98 & 0,0476 & 0,0011 & 0,0905 & 0,0027 & 0,01369 & 0,00029 & 0,00415 & 0,00033 & 0,7 & 7,6 & 1,8 & 92,4 & 2,6 & 87,6 & ,8 & 5 \\
\hline Zircon_13 & 740 & 830 & 1,12 & 0,0489 & 0,002 & 0,0927 & 0,003 & 0,01359 & 0,0004 & 0,00422 & 0,00032 & 0,83 & & 2,6 & 94,5 & 3 & 87 & 6 & 7 \\
\hline Zircon_16 & no value & no value & & 0,0460809 & 0,0000048 & 0,0989 & 0,0018 & & 0,00031 & & 0,00021 & 0,97 & & 2 & 100,5 & 7 & & 2 & 5 \\
\hline Zircon_17 & 529 & 862 & 1,63 & & & & & & & & & 0,96 & & 2,5 & 101,9 & 2,9 & & 2,5 & 7 \\
\hline Zircon_18 & 508 & 397 & 0,78 & & 0,0014 & 0,0933 & 0,0019 & & 0,00031 & & 0,00052 & 0,88 & & 2 & & 8 & 89,1 & 2 & 6 \\
\hline Zircon_21 & 1092 & 8540 & 7,82 & 0,0478 & 0,0012 & 0,0887 & 0,0015 & 0,0133 & 0,00021 & 0,00398 & 0,00021 & 0,69 & 85,18 & 1,4 & 90,6 & 1,4 & 05,2 & 1,4 & 5 \\
\hline Zircon_22 & 116,7 & 140,3 & 1,2 & 0,0460761 & 0,0000027 & 0,1195 & 0,0062 & 0,01791 & 0,00097 & 0,00431 & 0,0009 & 1 & 114,4 & 6,1 & 120,3 & 6 & 114 &, 1 & 4 \\
\hline Zircon_23 & 372,5 & 787 & 2,11 & 0,046095 & 0,000016 & 0,0986 & 0,0017 & 0,01482 & 0,00033 & 0,00424 & 0,00035 & 0,99 & 94,8 & 2,1 & 100,7 & 1,8 & 94,8 & 1 & 5 \\
\hline Zircon_25 & 526 & 561 & 1,07 & 0,04643 & 0,00046 & 0,0978 & 0,0017 & 0,01469 & 0,00026 & 0,0049 & 0,00025 & 0,96 & 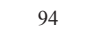 & 1,6 & 99,5 & 1,6 & 94 & 1,6 & 5 \\
\hline Zircon_26 & 974 & 875 & 0,9 & 0,0486 & 0,0019 & 0,0914 & 0,002 & 0,01366 & 0,0003 & 0,00424 & 0,00043 & 0,61 & 07,4 & 1,9 & 93,2 & 1,9 & 87,4 & 1,9 & 6 \\
\hline Zircon_27 & 356 & 1170 & 3,29 & 0,04625 & 0,00031 & 0,0929 & 0,0029 & 0,01398 & 0,00045 & 0,00416 & 0,00023 & 0,98 & 89,5 & 2,9 & 94,7 & 2,8 & 89,5 & 2,9 & 5 \\
\hline Zircon_28 & 187,6 & 2394 & 12,76 & 0,046099 & 0,000043 & 0,1011 & 0,0025 & 0,01512 & 0,00045 & 0,00405 & 0,0002 & 0,99 & 96,7 & 2,9 & 102,6 & 2,5 & 96,7 & 2,9 & 5 \\
\hline Zircon_29 & 290 & 2774 & 9,57 & 0,0460794 & 0,0000064 & 0,0957 & 0,0025 & 0,01437 & 0,00041 & 0,00385 & 0,00028 & 1 & 91,9 & 2,6 & 97,5 & 2,4 & 91,9 & 2,6 & 5 \\
\hline Zircon_30 & 912 & 699 & 0,77 & 0,0488 & 0,0017 & 0,0939 & 0,0019 & 0,01383 & 0,00022 & 0,00401 & 0,00026 & 0,65 & 88,5 & 1,4 & 95,7 & 1,8 & 88,5 & 1,4 & 7 \\
\hline Zircon_32 & 158,2 & 474 & 3 & 0,0460832 & 0,0000053 & 0,1018 & 0,0028 & 0,01526 & 0,00047 & 0,00408 & 0,00041 & 1 & 97,6 & 3 & 103,3 & 2,7 & 97,6 & 3 & 5 \\
\hline Zircon_33 & 573 & 3170 & 5,53 & 0,0478 & 0,0012 & 0,0885 & 0,0016 & 0,01329 & 0,00029 & 0,00396 & 0,00028 & 0,91 & 85,1 & 1,8 & 90,4 & 1,6 & 85,1 & 1,8 & 5 \\
\hline Zircon_37 & 111,6 & 327 & 2,93 & 0,0460785 & 0,0000013 & 0,0996 & 0,0034 & 0,01493 & 0,00056 & 0,0039 & 0,00031 & 1 & 95,6 & 3,6 & 101,2 & 3,3 & 95,6 & 3,6 & 5 \\
\hline Zircon_38 & 813 & 514 & 0,63 & 0,0484 & 0,0016 & 0,0922 & 0,0018 & 0,01365 & 0,00027 & 0,00425 & 0,00042 & 0,82 & 87,4 & 1,7 & 94 & 1,7 & 87,4 & 1,7 & 7 \\
\hline Zircon_39 & 153 & 464 & 3,03 & 0,0460892 & 0,0000098 & 0,0999 & 0,0044 & 0,01497 & 0,00064 & 0,00419 & 0,00034 & 0,99 & 95,8 & 4,1 & 101,5 & 4,3 & 95,8 & 4,1 & 5 \\
\hline Zircon_40 & 445 & 503,2 & 1,13 & 0,04622 & 0,00015 & 0,0883 & 0,0018 & 0,01318 & 0,00029 & 0,00386 & 0,00029 & 0,98 & 84,4 & 1,9 & 90,2 & 1,7 & 84,4 & 1,9 & 6 \\
\hline Zircon_41 & 110,4 & 52 & 0,47 & 0,0460733 & 0,0000016 & 0,0906 & 0,0041 & 0,01358 & 0,00063 & 0,0021 & 0,0012 & 1 & 86,9 & 4 & 92,4 & 4 & 86,9 & 4 & 5 \\
\hline
\end{tabular}

1: U and Th concentrations are calculated employing an external standard zircon as in Paton et al, 2010, Geochemistry, Geophysics, Geosystems, 2: 2 sigma uncertainties propagated according to Paton et al, 2010, Geochemistry, Geophysics, Geosystems,207Pb/206Pb ratios, ages and errors are calculated according to Petrus and Kamber, 2012, Geostandards Geoanalytical Research, Analyzed spot is 23 micrometer, using an analytical protocol modified from Solari et al, 2010, Geostandards Geoanalytical Research, Data measured employing an Xseries II ICPMS coupled to a Resonetics, Resolution M050 excimer laser workstation. 

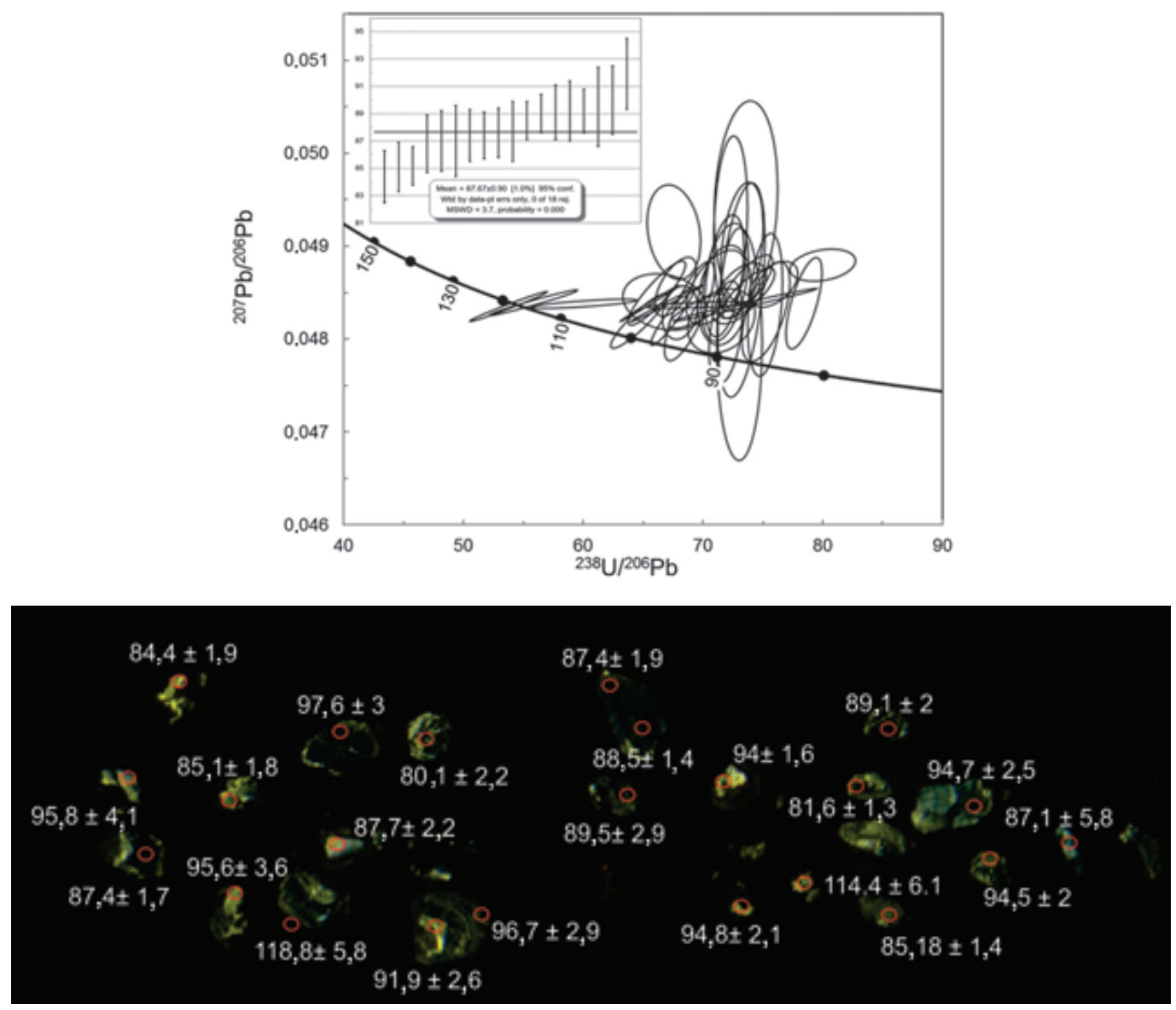

FIGURA 7. Edades U-Pb según diagrama de Tera-Wasserburg, media ponderada e imágenes de CL.

\section{INTERPRETACIÓN Y DISCUSIÓN}

Las secuencias de basaltos almohadillados intercalados con rocas piroclásticas en la región de Altamira, e incluidas en la formación Barroso presentan afinidad geoquímica de E-MORB y son similares a datos reportados en otros segmentos de la Cordillera Occidental, así como para otros segmentos correlacionados con un ambiente de pluma mantélica que se asocia a la formación de la Placa del Caribe en una región suroccidental en el Pacífico (Nivia, 1996; Kerr et al., 1997, 2003; Rodríguez y Arango, 2013; Villagómez y Spikings, 2013).

La edad de las rocas volcánicas de la Fm. Barroso en este trabajo no pudo ser obtenida por el método U-Pb, debido a la ausencia de circones en las muestras de basaltos recolectadas, sin embargo según la edad del Gabro de Altamira, la edad mínima de las secuencias volcánicas es de aproximadamente $88 \mathrm{Ma}$. Rocas intrusivas en la Formación Barroso como el Stock de Buriticá el cual se asocia a un origen de fusión parcial de la cuña del manto bajo el plateau oceánico
(Weber et al., 2015), presenta edades de $100 \mathrm{Ma}$ (Weber et al., 2015) y el Batolito de Sabanalarga de $85 \mathrm{Ma}$ (Ordóñez-Carmona et al., 2003), concordantes con datos bioestratigráficos para la Fm. Barroso donde se han obtenido edades entre el BarremianoAptiano (González, 2001) hasta el Aptiano-Albiano (Etayo-Serna, 1989), restringiendo la formación del plateau al Cretácico Inferior (FIGURA 9A). Otras secuencias clásticas discordantes sobre las unidades volcánicas presentan, edades más jóvenes, hasta el Maastrichtiano (Moreno-Sánchez and Pardo-Trujillo, 2003), que por su carácter cuarzoso y micáceo podrían marcar el momento de acreción del terreno oceánico a la margen continental.

Las edades heredadas en circones del Gabro de Altamira de 95 y $114 \mathrm{Ma}$, son igualmente compatibles con la generación de una provincia volcánica con afinidad de plateau oceánico, presente en la Cordillera Occidental, durante el Cretácico Inferior.

Recientemente Rodríguez y Arango (2013) sugirieren que las rocas de afinidad de plateau oceánico en la 
parte norte de la Cordillera Occidental, incluyendo el área de estudio, estarían localizadas hacia el eje y segmento occidental de la Cordillera Occidental. Sin embargo, los resultados geoquímicos presentados en este trabajo en la sección de Altamira sugieren que las secuencias basálticas con afinidad de plateau se extienden también a la parte oriental de la Cordillera Occidental.

Por otro lado, el Gabro de Altamira, cuerpo que intruye la Fm. Barroso presenta características de arco toleítico con anomalías negativas moderadas de $\mathrm{Ta}, \mathrm{Nb}$ y $\mathrm{Ti}$, además de enriquecimientos en Th y Zr. En este trabajo el Gabro de Altamira arrojó una edad de cristalización $\mathrm{U}-\mathrm{Pb}$ de $87,67 \pm 0,90 \mathrm{Ma}$.

Valores de $\varepsilon \mathrm{Nd}_{(\mathrm{T})}=4,78-6,58$ y relaciones ${ }^{87} \mathrm{Sr} /{ }^{86} \mathrm{Sr}=$ 0,70393 y 0,70348 (Ordóñez-Carmona, 2001) para el Gabro de Altamira (FIGURA 6F) son similares a otras rocas gabroicas y tonalíticas intrusivas en las secuencias volcánicas de la Cordillera Occidental como el Batolito de Sabanalarga y el Gabro de Hispania (OrdóñezCarmona, 2001; Weber et al., 2015).

La edad del Gabro de Altamira es similar a la de otros cuerpos que intruyen corteza oceánica con afinidad de Plateau Oceánico como el Batolito de Sabanalarga ( 85 a 98 Ma) (González et al., 1978; Weber et al., 2011; Ordóñez-Carmona et al., 2003), Batolito de Buga de 95 a $100 \mathrm{Ma}$ (Villagómez et al., 2011; Spikings et al., 2015), Tonalita de Buriticá de 91 a 100 Ma (Gobel and Stibane, 1979; Weber et al., 2015), Granito Pujilí en Ecuador de 85 Ma (Vallejo et al., 2006) y Batolito de Aruba en Aruba de alrededor 89 Ma (Van der Lelij et al., 2010), sugiriendo por lo tanto que un evento magmático plutónico con posibles equivalentes volcánicos asociados con afinidad de arco (FIGURAS 8 y 9B) como lo sugieren algunas de rocas volcánicas caracterizadas geoquímicamente al norte de la región de Altamira en la Cordillera Occidental (Rodríguez y Arango, 2013).

Weber et al. (2015) compara los patrones de elementos de REE, valores de $\varepsilon \mathrm{Nd}$ y relaciones ${ }^{87} \mathrm{Sr} /{ }^{86} \mathrm{Sr}$ del Batolito de Sabanalarga, Batolito de Buga, Tonalita de Buriticá y el Gabro de Altamira, sugiriendo que el comportamiento de estas unidades es muy similar. Además valores $\varepsilon \mathrm{Nd}$ y relaciones ${ }^{87} \mathrm{Sr} /{ }^{86} \mathrm{Sr}$ reportados para el Gabro de Altamira (Ordóñez-Carmona, 2001) son similares a las obtenidas para el Batolito de Aruba y el Granito Pujilí (Kerr et al., 1997; Weber et al., 2015), siendo estos últimos un poco más radiogénicos, sugiriendo una fuente primitiva donde el influjo de material radiogénico de posible afinidad continental siálica es limitado.

El magmatismo tipo Gabro de Altamira, además de las edades y relaciones isotópicas mencionadas anteriormente tiene una posición estructural y características petrológicas que hacen posible su correlación con cuerpos de gabros ubicados al sur como el Gabro de Hispania (Ordóñez-Carmona, 2001), así como a las facies gabróicas presentes en el Batolito de Sabanalarga o Gabro de Santa Fe (Nivia y Gómez-Tapias, 2005; Weber et al., 2011, 2015), sugiriendo por su carácter básico que este magmatismo formado sobre la corteza oceánica de tipo plateau oceánico debió tener una importante participación mantélica como es común en las fases tempranas de la subducción donde el flujo del manto astenosférico es primordial (Whattam and Stern, 2011).

Los cuerpos y diques intrusivos de composición andesítica encontrados en la zona de estudio intruyen ambas unidades (Fm. Barroso y Gabro de Altamira) y presentan características geoquímicas típicas de arco calco-alcalino (fuertes anomalías negativas de $\mathrm{Ta}, \mathrm{Nb}$ y Ti, enriquecimientos en Th y Zr), además de una edad $\mathrm{U}-\mathrm{Pb}$ en un grano de circón de una muestra de andesita porfídica de $86,9 \pm 4$ Ma (Zapata et al., 2013). Si bien más resultados geocronológicos son necesarios para validar esta edad, lo más razonable sería relacionar esta edad con edades reportadas para el Batolito de Sabanalarga (González et al., 1978; Weber et al., 2011). Sin embargo hay que recordar que en la zona se encuentran rocas andesíticas y dacíticas de edad Neógena y la edad obtenida podría ser una edad heredada (Jaramillo, 1976; González, 2001). Una edad Cretácica para las andesitas sugiere que la historia magmática de arco evolucionaría con la intrusión de las rocas andesíticas a características más calco-alcalinas después de la intrusión del Gabro de Altamira, conforme con un modelo de maduración del arco magmático (Whattam and Stern, 2011).

El plutonismo superpuesto al vulcanismo de plateau en los elementos acrecionados a la Placa del Caribe como los presentes en la Cordillera Occidental colombiana han sido atribuidos por varios autores a proceso de fusión parcial del plateau por interacción con la placa Suramericana (Nivia, 1987, 1989; Pindell and Kennan, 2009; Van der Lelij et al., 2010), después de acrecionado a la margen continental (Rodríguez y Arango, 2013). 
Igualmente esta podría corresponder a la conformación de una nueva zona de subducción, cuya vergencia aún se encuentra en discusión, pero que según algunos autores buzaría hacia el oeste (Thompson et al., 2004; Vallejo et al., 2006; Villagómez et al., 2011; Wright and Wyld, 2011; Weber et al., 2015) o a una continua evolución tectono-magmática de la Pluma-Arco (Whattam and Stern, 2015).

\section{$88 \mathrm{Ma}$}

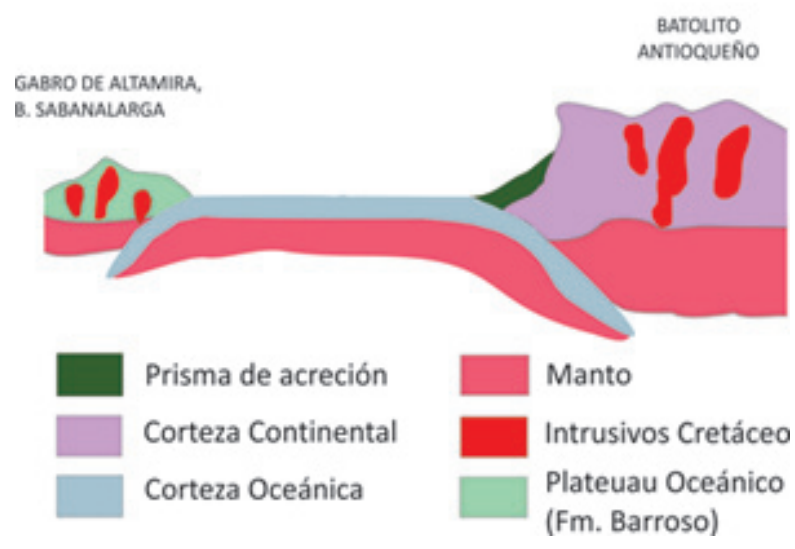

FIGURA 8. Modelo tectónico a 88 Ma para el margen noroccidental de la Placa Suramericana.

Los resultados presentados en este trabajo sobre el Gabro de Altamira, su correlación con cuerpos gabróicostoleíticos del Cretácico Tardío con afinidad de arco como ya fue mencionado es más afín con un modelo de arco calco-alcalino formado hacia el occidente con participación del manto y muy poca influencia de material radiogénico de la margen continental suramericana (FIGURAS 8 y 9A).

La acreción de este elemento oceánico a la margen continental habría ocurrido en el Cretácico superior-Paleoceno como lo sugiere el análisis termocronológico de la historia de enfriamiento en la Cordillera Occidental y Central (Villagómez y Spikings, 2013), la presencia de rocas clásticas con procedencia más siálica (alto contenido de cuarzo y moscovita) cubriendo las rocas oceánicas, además de los cambios en la sedimentación en el Cretácico Superior en el Valle del Magdalena, localizado al oriente de la Cordillera Central. El ambiente de estas unidades sedimentarias cretácicas varía de plataforma tranquila a ambientes deltaicos, y la procedencia de estos sedimentos, correspondería al basamento expuesto al occidente en la Cordillera Central (Villamil, 1999; Villagómez y Spikings, 2013).

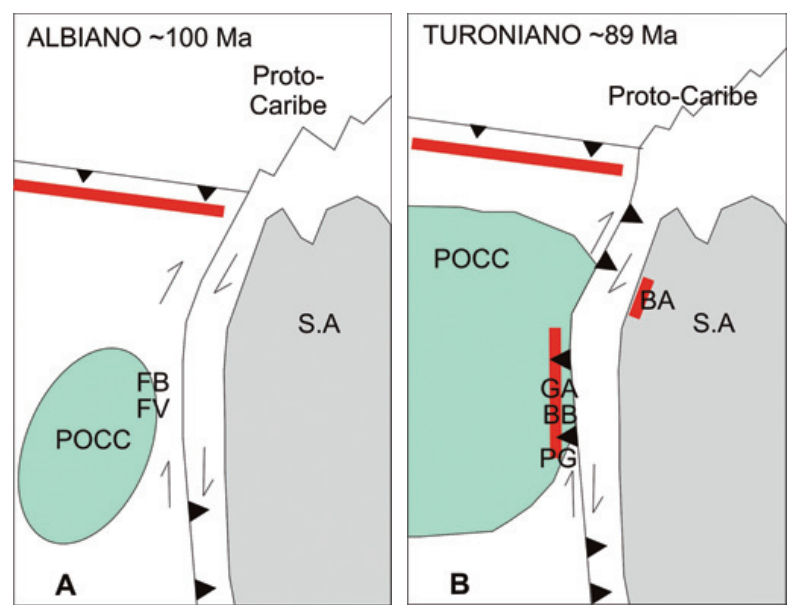

FIGURA 9. Modelo de evolución propuesto para la secuencia de roca básicas de Altamira (Modificado de Wright and Wyld, 2011). A. Albiano $\sim 100$ Ma. B. Turoniano 29 Ma. GA: Gabro de Altamira, AB: Batolito de Aruba, BA: Batolito Antioqueño, BB: Batolito de Buga, FB: Formación Barroso, FV: Formación volcánica, POCC: Plateau Oceánico Colombia-Carib, PG: Granito Pujilí.

\section{CONCLUSIONES}

Las secuencias volcánicas básicas de la Fm. Barroso constituidas por basaltos, diabasas e intercalados hacia el tope con rocas piroclásticas básicas presentan una afinidad geoquímica de E-MORB, correlacionable con otras rocas que conforman la Cordillera Occidental colombiana y ecuatorial, así como el Circum-Caribe, las cuales se asociarían a un ambiente de plateau oceánico.

La temporalidad para la formación de estas rocas volcánicas básicas, si bien todavía precisa de un detalle mayor, sugiere a partir de consideraciones paleontológicas y relaciones intrusivas que la formación del plateau oceánico que conforma el occidente colombiano tuvo lugar fundamentalmente en el Cretácico Inferior.

El Gabro de Altamira es un cuerpo intrusivo en la Fm. Barroso con edades de cristalización U-Pb de $87,67 \pm 0,90 \mathrm{Ma}$, y que presenta características de arco toleítico como anomalías negativas de $\mathrm{Nb}$, $\mathrm{Ta}$, Ti y enriquecimientos de $\mathrm{Th} / \mathrm{Yb}$ sugiriendo un componente de subducción (Pearce, 2008).

Las relaciones isotópicas existentes $\varepsilon \mathrm{Nd}_{(\mathrm{T})}=4,78-6,58$ y relaciones ${ }^{87} \mathrm{Sr} /{ }^{86} \mathrm{Sr}=0,70393$ y 0,70348 (OrdóñezCarmona, 2001) y la geocronología del Gabro de Altamira son similares a cuerpos plutónicos que intruyen la Cordillera Occidental en Colombia como el Batolito de Sabanalarga y el Gabro de Hispania, además 
del Batolito de Aruba en Aruba y el Granito Pujilí en Ecuador.

El Gabro de Altamira y los cuerpos correlacionables harían parte de un arco de islas oceánico con vergencia hacia occidente del dominio volcánico de afinidad plateau.

\section{AGRADECIMIENTOS}

En este trabajo se agradece especialmente al Dr. Luigi Solari y el Dr. Carlos Ortega de la UNAM por su ayuda para el análisis de las muestras en el ICP-MS y facilitar los laboratorios para la preparación de las muestras, a Ricardo Milián y José Duque por la ayuda para preparación de las muestras en el laboratorio. Un agradecimiento especial a Reinaldo Brito por ceder algunas muestras de litogeoquímica para la realización de este proyecto y a Teresa Orozco por su ayuda en las discusiones de los análisis geoquímicos. A Ricardo Ramírez por su compañía en el trabajo de campo y el envió de las muestras para los análisis.

\section{REFERENCIAS}

Álvarez, E., y González, H. 1978. Geología y geoquímica del Cuadrángulo I-7 (Urrao). INGEOMINAS. Informe 1761. 347 p. Mapa escala 1:100.000. Medellín. En: González, H., 2001. Mapa geológico del Departamento de Antioquia, escala 1:400.000. Memoria Explicativa. Ingeominas, pp. 167-170.

Amante, C., and Eakins, B.W. 2009. ETOPO1 1 ArcMinute Global Relief Model: Procedures, Data Sources and Analysis. NOAA Technical Memorandum NESDIS NGDC-24. National Geophysical Data Center, NOAA. Consultado el 3 de septimbre de 2016. https://www. ngdc.noaa.gov/mgg/image/color_etopo1_ice_low.jpg

Aspden, J.A. 1984. The geology of the western cordillera, Department of Valle, Colombia. CaliColombia. INGEOMINAS, pp.261, 278, 280, 299.

Barrero, D. 1979. Geology of the central Western Cordillera, west of Buga and Roldanillo, Colombia. Publicación Geológica Especial, INGEOMINAS, 4: 1-75.

Cardona, A., Valencia, V., Bustamante, C., GarcíaCasco, A., Ojeda, G., Ruiz, J., Saldarriaga, M., and Weber, M. 2010. Tectonomagmatic setting and provenance of the Santa Marta Schists, northern Colombia: Insights on the growth and approach of Cretaceous Caribbean oceanic terranes to the South American continent. Journal of South American Earth Sciences, 29(4): 784-804.
Case, J.E., Duran, L.G., Lopez, A., and Moore, W. 1971. Tectonic investigations in western Colombia and eastern Panama. Geological Society of America Bulletin, 82(10): 2685-2712.

Cediel, F., Shaw, R.P., and Cáceres, C. 2003. Tectonic assembly of the Northern Andean Block. In: Bartolini, C., Buffler, R.T., and Blickwede, J. (Eds). The CircumGulf of Mexico and the Caribbean: Hydrocarbon habitats, basin formation, and plate tectonics. AAPG Memoir 79, pp. 815-848.

Condie, K.C., and Abbott, D.H. 1999. Oceanic plateaus and hotspot islands: Identification and role in continental growth. Lithos, 46(1): 1-4.

Duque-Caro, H. 1984. Structural style, diapirism, and accretionary episodes of the Sinú-San Jacinto terrane, southwestern Caribbean borderland. Geological Society of America Memoirs, 162: 303-316.

Duque-Caro, H. 1989. El Arco de Dabeiba: Nuevas aportaciones al conocimiento del noroccidente de la Cordillera Occidental. V Congreso Colombiano de Geología, Bucaramanga, Colombia. Memorias I, pp. 108-126.

Etayo-Serna, F. 1989. Campanian to Maastrichtian fossils in the Northeastern Western Cordillera Colombia. Geología Norandina, 11: 23-31.

Feininger, T., Barrero, D., y Castro, N. 1972. Geología de parte de los departamentos de Antioquia y Caldas (Sub zona IIB). Boletín Geológico, 20: 1-173. INGEOMINAS, Bogotá.

Frey, M., De Capitani, C., and Liou, J.G. 1991. A new petrogenetic grid for low-grade metabasites. Journal of Metamorphic Geology, 9(4): 497-509.

Geoestudios-Ingeominas. 2005. Complementación geológica, geoquímica y geofísica de la parte occidental de las planchas 130 Santa Fé de Antioquia y 146 Medellín Occidental. Escala: 1:100.000.

Gobel, V., and Stibane, F. 1979. Edad K-Ar en hornblendas de plutones tonalíticos, Cordillera Occidental, Colombia, S.A. Publicación Especial de Geología, Universidad Nacional de Colombia, 17: 1-2.

González, H., Restrepo, J.J., Toussaint, J., y Linares, E. 1978. Edad radiométrica K/Ar del Batolito de Sabanalarga. Universidad Nacional de Colombia, Publicaciones Especiales Geol., 8: 1-4. 
González, H. 2001. Mapa geológico del departamento de Antioquia, Escala 1:400.000. Memoria explicativa. INGEOMINAS, Medellín.

Greene, A., Scoates, J., Weis, D., Katvala, E., Israel, S., and Nixon, G. 2010. The architecture of oceanic plateaus revealed by the volcanic stratigraphy of the accreted Wrangellia oceanic plateau. Geosphere, 6(1): 47-73.

Hauff, F., Hoernle, K., Van den Bogaard, P., Alvarado, G., and Garbe-Schönberg, D. 2000. Age and geochemistry of basaltic complexes in western Costa Rica: Contributions to the geotectonic evolution of Central America. Geochemistry, Geophysics, Geosystems, 1(5).

Hoernle, K., Hauff, F., and Van den Bogaard, P. 2004. 70 m.y. history (139-69 Ma) for the Caribbean large igneous province. Geology, 32(8): 697-700.

Jaramillo, J.M. 1976. Volcanic rocks of the río Cauca Valley, Colombia S.A. M.A. Thesis, Rice University, Houston.

Kerr, A.C., Marriner, G.F., Tarney, J., Nivia, A., Saunders, A.D., Thirlwall, M.F., and Sinton, C.W. 1997. Cretaceous basaltic terranes in Western Colombia: Elemental, chronological and $\mathrm{Sr}-\mathrm{Nd}$ isotopic constraints on petrogenesis. Journal of Petrology, 38(6): 667-702.

Kerr, A.C., White, R.V., Thompson, P.M.E., Tarney, J., and Saunders, A.D. 2003. No oceanic plateau-no Caribbean plate? The seminal role of an oceanic plateau in Caribbean plate evolution. In: Bartolini, C., Buffler, R.T., and Blickwede, J. (Eds.). The Circum-Gulf of Mexico and the Caribbean: Hydrocarbon habitats, basin formation, and plate tectonics. AAPG Memoir, 79: 126-168.

Kerr, A.C., Pearson, D.G., and Nowell, G.M. 2009. Magma source evolution beneath the Caribbean oceanic plateau: New insights from elemental and $\mathrm{Sr}-\mathrm{Nd}-\mathrm{Pb}-$ Hf isotopic studies of ODP Leg 165 Site 1001 basalts. Geological Society, London, Special Publications, 328(1): 809-827.

Lozano-Santacruz, R., Verma, S.P., Girón, P., VelascoTapia, F., Morán-Zenteno, D., Viera, F., y Chávez, G. 1995. Calibración preliminar de fluorescencia de rayos $\mathrm{X}$ para análisis cuantitativo de elementos mayores en rocas ígneas. Actas INAGEQ, 1: 203-208

Ludwig, K. 2004. Isoplot/Ex, ver. 3. A geochronological toolkit for Microsoft Excel. Berkeley Geochronology Center, California. Publication núm. 4.
Maya, M., y González, H. 1995. Unidades litodémicas en la Cordillera Central de Colombia. Boletín Geológico, Ingeominas, 35(2-3): 43-57.

McPhie, J., Doyle, M., and Allen, R. 1993. Volcanic textures: A guide to the interpretation of texture in volcanic rocks. University of Tasmania. 1-140 p.

Meissner, R.O., Flueh, E.R., Stibane, F., and Berg, E. 1976. Dynamics of the active plate boundary in southwest Colombia according to recent geophysical measurements. Tectonophysics, 35(1-3): 115-136.

Mejía, M., Álvarez, E., y González, H. 1983. Mapa Geológico de la Plancha 130 (Santafé de Antioquia). Escala 1:100.000. INGEOMINAS.

Meschede, M., and Frisch, W. 1998. A plate-tectonic model for the Mesozoic and Early Cenozoic history of the Caribbean plate. Tectonophysics, 296(3-4): 269-291.

Moreno-Sánchez, M., and Pardo-Trujillo, A. 2003. Stratigraphical and sedimentological constraints on western Colombia: Implications on the evolution of the Caribbean Plate. In: Bartolini, C., Buffler, R.T., and Blickwede, J. (Ed.). Circum-Gulf of Mexico and the Caribbean: Hydrocarbon habitats, basin formation, and plate tectonics. AAPG Memoir 79, Chapter 40, pp. 891-924.

Mori, L., Gómez-Tuena, A., Cai, Y., and Goldstein, S. 2007. Effects of prolonged flat subduction on the Miocene magmatic record of the central Trans-Mexican Volcanic Belt. Chemical Geology, 244(3-4): 452-473.

Nakamura, N. 1974. Determination of REE, Ba, Fe, $\mathrm{Mg}$, Na and $\mathrm{K}$ in carbonaceous and ordinary chondrites. Geochimica et Cosmochimica Acta, 38(5), 757-775.

Nivia, A. 1987. The geochemistry and origin of the Amaime and Volcanic sequences, SW Colombia. M.Phil. Thesis, University of Leicester, UK. 164p.

Nivia, A. 1989. El Terreno Amaime-Volcánica una provincia acrecionada de basaltos de meseta oceánica. V Congreso Colombiano de Geología, Bucaramanga, Colombia. Memorias I, pp. 1-30.

Nivia, A. 1996. The Bolivar mafic-ultramafic complex, SW Colombia: the base of an obducted oceanic plateau. Journal of South American Earth Sciences, 9(1-2): 59-68.

Nivia, A., y Gómez-Tapias, J. 2005. El Gabro Santa Fe de Antioquia y la Cuarzodiorita Sabanalarga, una propuesta de nomenclatura litoestratigráfica para dos 
cuerpos plutónicos diferentes agrupados previamente como Batolito de Sabanalarga en el Departamento de Antioquia, Colombia. X Congreso Colombiano de Geología, pp 1-9.

Ordóñez-Carmona, O. 2001. Caracterização isotópica $\mathrm{Rb} / \mathrm{Sr}$ e $\mathrm{Sm} / \mathrm{Nd}$ dos principais eventos magmáticos nos Andes Colombianos. Tesis de Doctorado, Universidad de Brasília, Brasil. 177p.

Ordóñez-Carmona, O., Pimentel, M.M., y Abad-Posada, A. 2003. Batolito de Sabanalarga: Magmatismo de arco o de pluma?. IX Congreso Colombiano de Geología, Medellín, Colombia.

Parra, E. 1978. Estudio geológico de las rocas básicas y sedimentarias en Giraldo, Antioquia. TDG. Facultad de Minas. Universidad Nacional de Colombia. Medellín. 90 p.

Pearce, J.A. 2008. Geochemical fingerprinting of oceanic basalts with applications to ophiolite classification and the search for Archean oceanic crust. Lithos, 100(1-4): 14-48.

Peccerillo, A., and Taylor, S.R. 1976. Geochemistry of eocene calc-alkaline volcanic rocks from Kastamonu area, Northern Turkey. Contributions to Mineralogy and Petrology, 58(1): 63-81.

Pindell, J., Kennan, L., Maresch, W.V., Stanek, K., Draper, G., and Higgs, R. 2005. Plate-kinematics and crustal dynamics of circum-Caribbean arc-continent interactions: Tectonic controls on basin development in Proto-Caribbean margins. Special Paper Geological Society of America, 394: 7-52.

Pindell, J., and Kennan, L. 2009. Tectonic evolution of the Gulf of Mexico, Caribbean and northern South America in the mantle reference frame: An update. In: James, K.H., Lorente, M.A., and Pindell, J.L. (Eds.). The geology and evolution of the region between North and South America. Geological Society of London Special Publication, 328:1-55.

Ramírez, J. 1976. Estudio geológico de la secuencia de rocas básicas de Altamira. Tesis. Facultad de Minas, Universidad Nacional de Colombia, Sede Medellín. 108p.

Restrepo, J.J.y Toussaint, J.F. 1976. Edades radiométricas de algunas rocas de Antioquia. Publicación Especial Geológica, Facultad de Minas, Universidad Nacional de Colombia, Medellín, (6):1-15.
Restrepo, J.J., and Toussaint, J.F. 1988. Terranes and Continental accretion in the Colombian Andes. Episodes, 11(3): 189-193.

Restrepo, J.J., Ordóñez-Carmona, O., Martens, U., y Correa, A.M. 2009. Terrenos, complejos y provincias en la Cordillera Central de Colombia. XII Congreso Colombiano de Geología, Paipa, Colombia, pp. 16.

Révillon, S., Arndt, N.T., Chauvel, C., and Hallot, E. 2000. Geochemical study of ultramafic volcanic and plutonic rocks from Gorgona Island, Colombia: The plumbing system of an oceanic plateau. Journal of Petrology, 41(7): 1127-1153.

Rodríguez, G., y Arango, M.I. 2013. Formación Barroso: Arco volcánico Toleitico y diabasas de San José de Urama: Un prisma acrecionario T-Morb en el segmento norte de la Cordillera Occidental de Colombia. Boletín Ciencias de la Tierra, 33: 17-38.

Rubatto, D. 2002. Zircon trace element geochemistry: distribution coefficients and the link between $\mathrm{U}-\mathrm{Pb}$ ages and metamorphism. Chemical Geology, 184: 123-138.

Serrano, L., Ferrari, L., López, M., Petrone, C., and Jaramillo, C. 2011. An integrative geologic, geochronologic and geochemical study of Gorgona Island, Colombia: Implications for the formation of the Caribbean Large Igneous Province. Earth and Planetary Science Letters, 309(3-4): 324-336.

Sláma, J., Košler, J., Condon, D., Crowley, J., Gerdes, A., Hanchar, J., Horstwood, M., Morris, G., Nasdala, L., Norberg, N., Schaltegger, U., Schoene, B., Tubrett, M., and Whitehouse, M. 2008. Plešovice zircon: A new natural reference material for $\mathrm{U}-\mathrm{Pb}$ and $\mathrm{Hf}$ isotopic microanalysis. Chemical Geology, 249(1-2): 1-35.

Sinton, C.W., Duncan, R.A., Storey, M., Lewis, J., and Estrada, J.J. 1998. An oceanic flood basalt province within the Caribbean Plate. Earth and Planetary Science Letters, 155(3-4): 221-235.

Solari, L., Gómez-Tuena, A., Bernal, J., Pérez-Arvizu, O., and Tanner, M. 2010 U-Pb zircon geochronology with an integrated LA-ICP-MS microanalytical workstation: achievements in precision and accuracy. Geostandards and Geoanalytical Research, 34(1): 5-18.

Spikings, R.A., Cochrane, R., Villagómez, D., Van der Lelij, R., Vallejo, C., Winkler, W., and Beate, B. 2015. The geological history of northwestern South America: 
from Pangaea to the early collision of the Caribbean Large Igneous Province (290-75 Ma). Gondwana Research, 27(1): 95-139.

Sun, S., and McDonough, W. 1989. Chemical and isotropic systematics of oceanic basalts: implications for mantle composition and processes. In: Saunders, A.D., and Norry, M.J. (eds). Magmatism in the Ocean Basins. Geological Society Special Publication No. 42, pp. 313-345. Londres.

Tera, F., and Wasserburg, G. 1972. U-Th-Pb systematics in lunar highland samples from the Luna 20 and Apollo 16 missions. Earth and Planetary Science Letters, 17(1): $36-51$.

Thompson, P.M.E., Kempton, P.D., White, R.V., Saunders, A.D., Kerr, A.C., Tarney, J., and Pringle, M.S. 2004. Elemental, Hf-Nd isotopic and geochronological constraints on an island arc sequence associated with the Cretaceous Caribbean plateau: Bonaire, Dutch Antilles. Lithos, 74: 91-116.

Toussaint, J.F., y Restrepo, J.J. 1974. Obducción Cretácea en el Occidente Colombiano. Publicación Especial de Geología, Universidad Nacional de Colombia, Medellín, 3: 1-26.

Toussaint, J.F., y Restrepo, J.J. 1976. Modelos orogénicos de tectónica de placas en los Andes Colombianos. Boletín de Ciencias de la Tierra, 1: 1-47.

Toussaint, J.F., y Restrepo, J.J. 1978. Edad K/Ar de dos rocas básicas del flanco noroccidental de la Cordillera Central. Boletín de Ciencias de la Tierra, 14: 1-7.

Vallejo, C., Spikings, R.A., Luzieux, L., Winkler, W., Chew, D., and Page, L. 2006. The early interaction between the Caribbean Plateau and the NW South American Plate. Terra Nova, 18(4): 264-269.

Van der Lelij, R., Spikings, R.A., Kerr, A.C, Kounov, A., Cosca, M., Chew, D., and Villagómez, D. 2010. Thermochronology and tectonics of the Leeward Antilles: Evolution of the southern Caribbean Plate boundary zone. Tectonics, 29(6): 1-30.

Verma, S.P., Lozano-Santacruz, R., Girón, P., y Velasco-Tapia, F. 1996. Calibración preliminar de fluorescencia de rayos X para análisis cuantitativo de elementos traza en rocas ígneas. Actas INAGEQ, 2: 237-242.
Villagómez, D. 2010. Thermochronology, geochronology and geochemistry of the Western and Central cordilleras and Sierra Nevada de Santa Marta, Colombia: The tectonic evolution of NW South America. Ph.D. Thesis, Université de Genève. 142p.

Villagómez, D., Spikings, R.A., Magna, T., Kammer, A., Winkler, W., and Beltrán, A. 2011. Geochronology, geochemistry and tectonic evolution of the Western and Central cordilleras of Colombia. Lithos, 125(3-4): 875-896.

Villagómez, D., and Spikings, R.A. 2013. Thermochronology and tectonics of the Central and Western Cordilleras of Colombia: Early CretaceousTertiary evolution of the Northern Andes. Lithos, 160161: 228-249.

Villamil, T. 1999. Campanian-Miocene tectonostratigraphy, depocenter evolution and basin development of Colombia and western Venezuela. Palaeogeography, Palaeoclimatology, Palaeoecology, 153(1-4): 239-275.

Winchester, J.A., and Floyd, P.A. 1977. Geochemical discrimination of different magma series and their differentiation products using immobile elements. Chemical Geology, 20: 325-343.

Weber, M., Gómez-Tapias, J., Duarte, E., Cardona, A., and Vinazco, C. 2011. Geochemistry of the Santa Fé Batholith in NW Colombia-Remnant of an accreted Cretaceous arc. XIV Congreso Latinoamericano de Geología, Medellín, Colombia, pp. 128-129.

Weber, M., Gómez-Tapias, J., Cardona, A., Duarte, E., Pardo-Trujillo, A., and Valencia, V.A. 2015. Geochemistry of the Santa Fé Batholith and Buriticá Tonalite in NW Colombia - Evidence of subduction initiation beneath the Colombian Caribbean Plateau. Journal of South America Earth Science, 62: 257-274.

Whattam, S.A., and Stern, R.J. 2011. The 'subduction initiation rule': A key for linking ophiolites, intraoceanic forearcs and subduction initiation. Contributions to Mineralogy and Petrology, 162(5):1031-1045.

Whattam, S.A., and Stern, R.J. 2015. Late Cretaceous plume-induced subduction initiation along the southern margin of the Caribbean and NW South America: The first documented example with implications for the onset of plate tectonics. Gondwana Research, 27(1): 38-63. 
White, R.V., Tarney, J., Kerr, A.C., Saunders, A.D., Kempton, P.D., Pringle, M.S., and Klaver, G.T. 1999. Modification of an oceanic plateau, Aruba, Dutch Caribbean: Implications for the generation of continental crust. Lithos, 46(1): 43-68.

White, S., Trenkamp, R., and Kellogg, J.N. 2003. Recent crustal deformation and the earthquake cycle along the Ecuador-Colombia subduction zone. Earth and Planetary Science Letters, 216(3): 231-242.

Wright, J.E., and Wyld, S.J. 2011. Late Cretaceous subduction initiation on the eastern margin of the Caribbean-Colombian Oceanic Plateau: One Great Arc of the Caribbean. Geosphere, 7(2): 468-493.

Zapata, J.P., Restrepo, J.J., Martens, U., Cardona, A., and Brito, R. 2013. Geochronology and geochemistry of the basic sequence of Altamira, Antioquia, Western Cordillera of Colombia. XIV Congreso Colombiano de Geología, Bogotá, Colombia, pp. 375.

Trabajo recibido: octubre 19 de 2016

Trabajo aceptado: febrero 15 de 2017

Manuscrito publicado en internet: abril 06 de 2017 